\title{
Anomalies in $B$ mesons decays: a phenomenological approach
}

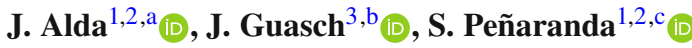 \\ ${ }_{1}^{1}$ Departamento de Física Teórica, Facultad de Ciencias, Universidad de Zaragoza, Pedro Cerbuna 12, 50009 Zaragoza, Spain \\ ${ }^{2}$ Centro de Astropartículas y Física de Altas Energías (CAPA), Universidad de Zaragoza, Zaragoza, Spain \\ ${ }^{3}$ Deptartament de Física Quàntica i Astrofísica and Institut de Ciències del Cosmos (ICCUB), Universitat de Barcelona, Martí i Franquès 1, 08028 Barcelona, \\ Catalonia, Spain
}

Received: 20 January 2021 / Accepted: 19 January 2022

(C) The Author(s) 2022

\begin{abstract}
The experimental measurements on flavour physics, in tension with Standard Model predictions, exhibit large sources of lepton flavour universality violation. We perform an analysis of the effects of the global fits on the Wilson coefficients assuming the Standard Model Effective Field Theory with semileptonic dimension six operators at $1 \mathrm{TeV}$, and by including a set of different scenarios in which the New Physics contributions to the Wilson coefficients are present in one, two or three of the Wilson coefficients at a time. We compare the results of the global fit with respect to two cases: the Standard Model and the more general case in which new physics modifies three independent Wilson coefficients. The last mentioned scenario is the favoured one for explaining the tension between Standard Model predictions and $B$-physics anomalies, but a specific more restricted scenario can provide similar goodness with a smaller set of free parameters. A discussion of the implications of our analysis in leptoquark models is included.
\end{abstract}

\section{Introduction}

In the last few years, many interesting measurements on flavour physics have been performed at the LHC [1-15], BaBar [16] and Belle [17-21]. Several experimental collaborations observed lepton flavour universality violating (LFUV) processes in $B$ meson decays that would be a clear sign for physics beyond the Standard Model (SM). Some of these decays allow us to build optimised observables, as ratios of these decays, that are theoretically clean observables and whose measurements are in tension with SM predictions. One example is the case of the $R_{D^{(*)}}$ ratios:

$$
R_{D^{(*)}}^{\ell}=\frac{\mathrm{BR}\left(B \rightarrow D^{(*)} \tau \bar{\nu}_{\tau}\right)}{\left[\operatorname{BR}\left(B \rightarrow D^{(*)} e \bar{v}_{e}\right)+\operatorname{BR}\left(B \rightarrow D^{(*)} \mu \bar{v}_{\mu}\right)\right] / 2},
$$

and

$$
R_{D^{(*)}}^{\mu}=\frac{\operatorname{BR}\left(B \rightarrow D^{(*)} \tau \bar{\nu}_{\tau}\right)}{\operatorname{BR}\left(B \rightarrow D^{(*)} \mu \bar{v}_{\mu}\right)} .
$$

In the $b \rightarrow c \ell v$ transitions, signs of violation of lepton universality have been observed only in the $e-\tau$ and $\mu-\tau$ cases, while the universality has been tested to great precision in the $e-\mu$ case [17,18,22]. As a consequence, both $R_{D^{(*)}}^{\ell}$ and $R_{D^{(*)}}^{\mu}$ should have similar predictions and measurements. Note that $R_{D}$ and $R_{D^{(*)}}$ ratios should only have similar predictions in the SM or any other lepton flavour universality (LFU) theory. The measured values of these ratios at BaBar, Belle and LHCb experiments are larger than the SM prediction $\left(R_{D}^{\ell \mathrm{SM}}=0.299 \pm 0.003, R_{D^{*}}^{\ell \mathrm{SM}}=R_{D^{*}}^{\mu \mathrm{SM}}=0.258 \pm 0.005\right.$ [23]). The first deviation was found by BaBar in 2012 [16]

$$
R_{D}^{\ell}=0.440 \pm 0.058 \pm 0.042, \quad R_{D^{*}}^{\ell}=0.332 \pm 0.024 \pm 0.018 .
$$

The latest experimental results from Belle are [20]

$$
R_{D}^{\ell}=0.307 \pm 0.037 \pm 0.016, \quad R_{D^{*}}^{\ell}=0.283 \pm 0.018 \pm 0.014
$$

and from $\mathrm{LHCb}[12]$

$$
R_{D^{*}}^{\mu}=0.291 \pm 0.019 \pm 0.026 \pm 0.013
$$

\footnotetext{
a e-mail: jalda@unizar.es

b e-mail: jaume.guasch@ub.edu

c e-mail: siannah@unizar.es (corresponding author)
} 
The combined result from the Belle measurements has a compatibility with the SM predictions of $1.2 \sigma$, much better than previous measurements of these observables (see, for example, a compatibility of $3.6 \sigma$ as of 2016 [24]). The world average of the experimental values for the $R_{D^{(*)}}$ ratios, as obtained by the Heavy Flavour Averaging Group (HFLAV), assuming universality in the lighter leptons, is [23]

$$
R_{D}^{\mathrm{ave}}=0.340 \pm 0.027 \pm 0.013, \quad R_{D^{*}}^{\mathrm{ave}}=0.295 \pm 0.011 \pm 0.008
$$

$R_{D}$ exceeds the SM value by $1.4 \sigma$ and $R_{D^{*}}$ by $2.5 \sigma$. When combined together, included their correlation, the excess is $3.08 \sigma$.

Another class of $B$ meson observables showing signs of LFUV is related to $b \rightarrow s \ell^{+} \ell^{-}$processes, namely the optimised angular observable $P_{5}^{\prime}[25]$ and the $R_{K^{(*)}}$ ratios,

$$
R_{K^{(*)}}=\frac{\mathrm{BR}\left(B \rightarrow K^{(*)} \mu^{+} \mu^{-}\right)}{\operatorname{BR}\left(B \rightarrow K^{(*)} e^{+} e^{-}\right)} .
$$

The $R_{K^{(*)}}$ ratios are observables that have small theoretical uncertainties, and in the $\mathrm{SM}, R_{K}=R_{K^{*}}=1$ with uncertainties of the order of $1 \%[26,27]$ as a consequence of LFU. The latest experimental results from LHCb, in the specified regions of $q^{2}$ di-lepton invariant mass, are

$$
\begin{aligned}
R_{K}^{[1.1,6]} & =0.846_{-0.039-0.012}^{+0.042+0.013}, \\
R_{K^{*}}^{[0.045,1.1]}=0.66_{-0.07}^{+0.11} \pm 0.03, \quad R_{K^{*}}^{[1.1,6]} & =0.69_{-0.07}^{+0.11} \pm 0.05 .
\end{aligned}
$$

The compatibility of the individual measurements with respect to the SM predictions is of $3.1 \sigma$ for the $R_{K}$ ratio, $2.3 \sigma$ for the $R_{K} *$ ratio in the low $-q^{2}$ region and $2.4 \sigma$ in the central- $q^{2}$ region. The Belle collaboration has also recently reported experimental results for the $R_{K^{(*)}}$ ratios [19,21], although with less precision than the LHCb measurements.

A great theoretical effort has been devoted to the understanding of the deviations in the $R_{K^{(*)}}$ observables [28-42], the deviations in the $R_{D^{(*)}}$ observables [22,43-58], and combined explanations for the deviations in $R_{K^{(*)}}$ and $R_{D^{(*)}}$ [59-74] and references therein. Besides, the experimental data have been used to constrain new physics (NP) models. Several global fits have been performed in the literature [75-81].

One of the most widely used tools to study any possible New Physics (NP) contribution that explains the above experimental results is the effective field theories. The effective Hamiltonian approach allows us to perform a model-independent analysis of NP effects. In this way, it is possible to obtain constraints on NP contributions to the Wilson coefficients of the Hamiltonian from the experimental results.

In this work, we investigate the effects of the global fits to the Wilson coefficients assuming a model-independent effective Hamiltonian approach and including a discussion of the consequences of our analysis in leptoquark models. We define different scenarios for the phenomenological study by considering the NP contributions to the Wilson coefficients in such a way that NP is present in one, two or three of the Wilson coefficients simultaneously. These scenarios are used to study the impact of the global fits on the Wilson coefficients and, therefore, to exhibit more clearly which combinations of Wilson coefficients are preferred and/or constrained by experimental data.

We begin in Sect. 2 by presenting a brief summary of the effective field theory used to describe possible NP contributions to $B$ decays observables. Then, Sect. 3 is devoted to the global fits to the Wilson coefficients, presenting the set of scenarios that we are going to analyse. As already explained, we will work in different scenarios that arise by considering the presence of NP contributions in one, two or three of the Wilson coefficients. We will compare the results of the global fit in each scenario with respect to two cases: the SM and the best-fit point of the three independent Wilson coefficients scenario (the most general case). This particular choice of the Wilson coefficients that will enter our analysis is the main difference with respect to previous global fits analysis in the recent literature. Section 3.1 is devoted to discuss in more detail the most general proposed scenario, Scenario VII, in which the prediction of the $R_{D^{(*)}}$ and $R_{K^{(*)}}$ observables is improved. Finally, the phenomenological implications of our analysis in leptoquark models are included in Sect. 4. Conclusions are presented in Sect. 5. Appendix A contains the list of observables that contribute to the global fit with their prediction in the most general scenario: the global fit to three independent Wilson coefficients receiving NP contributions.

\section{Effective field theories for $B$ observables}

One of the most widely used tools to study any possible NP contribution is the effective field theories. The Standard Model Effective Field Theory (SMEFT) is formulated at an energy scale $\mu_{\text {SMEFT }}=\Lambda$ higher than the electroweak scale, and the degrees of freedom are all the SM fields. The Weak Effective Theory (WET) is formulated at an energy scale below the electroweak scale, for example $\mu_{\mathrm{WET}}=m_{b}$, and the top quark, Higgs, $W$ and $Z$ bosons are integrated out.

In this work, all the numerical analyses will be performed using only the SMEFT operators, while the WET Lagrangian will be useful for the discussion of the results. 


\subsection{Weak Effective Theory}

The relevant terms of the WET Lagrangian [82-85] are

$$
\mathcal{L}_{\text {eff }}=-\frac{4 G_{F}}{\sqrt{2}} V_{c b} \sum_{\ell=e, \mu, \tau}\left(1+C_{V L}^{\ell}\right) \mathcal{O}_{V L}^{\ell}+\frac{4 G_{F}}{\sqrt{2}} V_{t b} V_{t s}^{*} \frac{e^{2}}{16 \pi^{2}} \sum_{\ell=e, \mu}\left(C_{9}^{\ell} \mathcal{O}_{9}^{\ell}+C_{10}^{\ell} \mathcal{O}_{10}^{\ell}\right),
$$

where $G_{F}$ is the Fermi constant, $e$ is the electromagnetic coupling, $V_{q q^{\prime}}$ are the elements of the Cabibbo-Kobayashi-Maskawa $(\mathrm{CKM})$ matrix and with the dimension-six operators defined as

$$
\mathcal{O}_{V L}^{\ell}=\left(\bar{c}_{L} \gamma_{\alpha} b_{L}\right)\left(\bar{\ell}_{L} \gamma^{\alpha} v_{\ell}\right), \quad \mathcal{O}_{9}^{\ell}=\left(\bar{s}_{L} \gamma_{\alpha} b_{L}\right)\left(\bar{\ell} \gamma^{\alpha} \ell\right), \quad \mathcal{O}_{10}^{\ell}=\left(\bar{s}_{L} \gamma_{\alpha} b_{L}\right)\left(\bar{\ell} \gamma^{\alpha} \gamma_{5} \ell\right),
$$

and their corresponding Wilson coefficients $C_{V L}^{\ell}, C_{9}^{\ell}$ and $C_{10}^{\ell}$. The $C_{9}^{\ell}$ and $C_{10}^{\ell}$ Wilson coefficients have contributions from the SM processes as well as any NP contribution:

$$
C_{i}^{\ell}=C_{i}^{\mathrm{SM} \ell}+C_{i}^{\mathrm{NP} \ell}, \quad i=9,10,
$$

whereas $C_{V L}^{\ell}$ only receives contributions from NP. In the present work, we analyse the NP contributions.

The $R_{D^{(*)}}$ ratios obey the following expression [61,64]:

$$
\begin{aligned}
& R_{D^{(*)}}^{\ell}=R_{D^{(*)}}^{\ell, \mathrm{SM}} \frac{\left|1+C_{V L}^{\tau}\right|^{2}}{\left(\left|1+C_{V L}^{e}\right|^{2}+\left|1+C_{V L}^{\mu}\right|^{2}\right) / 2}, \\
& R_{D^{(*)}}^{\mu}=R_{D^{(*)}}^{\mu, \mathrm{SM}} \frac{\left|1+C_{V L}^{\tau}\right|^{2}}{\left|1+C_{V L}^{\mu}\right|^{2}} .
\end{aligned}
$$

The dependence of the $R_{K^{*}}$ ratios on the Wilson coefficients has been previously obtained in [41], where an analytic computation of $R_{K^{* 0}}$ as a function of $C_{9}^{\mathrm{NP} \mu}, C_{10}^{\mathrm{NP} \mu}$ in the region $1.1 \leq q^{2} \leq 6.0 \mathrm{GeV}^{2}$ was performed. The result is given by [41]

$$
R_{K^{*}}^{[1.1,6]} \simeq \frac{0.9875+0.1759 \operatorname{Re} C_{9}^{\mathrm{NP} \mu}-0.2954 \operatorname{Re} C_{10}^{\mathrm{NP} \mu}+0.0212\left|C_{9}^{\mathrm{NP} \mu}\right|^{2}+0.0350\left|C_{10}^{\mathrm{NP} \mu}\right|^{2}}{1+0.1760 \operatorname{Re} C_{9}^{\mathrm{NP} e}-0.3013 \operatorname{Re} C_{10}^{\mathrm{NP} e}+0.0212\left|C_{9}^{\mathrm{NP} e}\right|^{2}+0.0357\left|C_{10}^{\mathrm{NP} e}\right|^{2}} .
$$

\subsection{Standard Model Effective Field Theory}

We consider NP contributions at an energy scale $\Lambda(\Lambda \sim \mathcal{O}(\mathrm{TeV}))$ described by the SMEFT Lagrangian as given in [86], where a complete list of the independent dimension-six operators that are allowed by the SM gauge symmetries is presented. The SMEFT Lagrangian is given by [86]

$$
\mathcal{L}_{\mathrm{SMEFT}}=\frac{1}{\Lambda^{2}}\left(C_{\ell q(1)}^{i j k l} O_{\ell q(1)}^{i j k l}+C_{\ell q(3)}^{i j k l} O_{\ell q(3)}^{i j k l}\right)
$$

where the dimension-six operators are defined as

$$
O_{\ell q(1)}^{i j k l}=\left(\bar{\ell}_{i} \gamma_{\mu} \ell_{j}\right)\left(\bar{q}_{k} \gamma^{\mu} q_{l}\right), \quad O_{\ell q(3)}^{i j k l}=\left(\bar{\ell}_{i} \gamma_{\mu} \tau^{I} \ell_{j}\right)\left(\bar{q}_{k} \gamma^{\mu} \tau^{I} q_{l}\right)
$$

$\ell$ and $q$ are the lepton and quark $S U(2)_{L}$ doublets defined in the mass basis, ${ }^{1} \tau^{I}$ the Pauli matrices, and $i, j, k, l$ denote generation indices. The $O_{\ell q(1)}$ operator couples two $S U(2)_{L}$-singlet currents, while the $O_{\ell q(3)}$ operator couples two $S U(2)_{L}$-triplet currents. Consequently, $O_{\ell q(1)}$ only mediates flavour-changing neutral processes and $O_{\ell q(3)}$ mediates both flavour-changing neutral and charged processes. We will restrict our analysis to operators including only third-generation quarks and same-generation leptons, and we will use the following notation for their Wilson coefficients:

$$
C_{\ell q}^{e} \equiv C_{\ell q}^{1133}, \quad C_{\ell q}^{\mu} \equiv C_{\ell q}^{2233}, \quad C_{\ell q}^{\tau} \equiv C_{\ell q}^{3333}
$$

This particular choice of the Wilson coefficients that will enter our analysis is motivated by the fact that the most prominent discrepancies between SM predictions and experimental measurements, namely $R_{K^{(*)}}$ and $R_{D^{(*)}}$, affect the third quark generation. From a symmetry point of view, this would amount to imposing an $U(2)^{3}=U(2)_{q} \times U(2)_{u} \times U(2)_{d}$ symmetry between the first and second quark generations [88-90], that remain SM-like. No restriction is imposed on the third quark generation. In the lepton sector, we only consider diagonal entries in order to avoid lepton flavour violating (LFV) decays. This flavour structure for NP contributions has been presented in [90] as a minimal working set-up.

These operators generate the $C_{V L}^{\ell}, C_{9}^{\ell}$ and $C_{10}^{\ell}$ operators of the electroweak effective field theory when matched at the electroweak scale $\mu_{\mathrm{EW}}$. Using the package wilson [91], we define the $C_{\ell q}$ operators at $\Lambda=1 \mathrm{TeV}$, we calculate their running down to

\footnotetext{
1 As given in [87], and used on the package wilson [91], in both the "Warsaw" basis and the "Warsaw mass" basis, the lepton and $d$-quark fields are defined so that their mass matrices are diagonal. Consequently, translating from one to another does not modify the Lagrangian in Eq. (14).
} 
$\mu_{\mathrm{EW}}=M_{Z}$, then match them with the WET operators and finally run the down to $\mu=m_{b}$, where the $B$-physics observables are computed. By taking the SMEFT Wilson coefficients at $\Lambda=1 \mathrm{TeV}$, we found the following relations between the Wilson coefficients at high and low energies:

$$
\begin{array}{ll}
C_{9}^{\mathrm{NP} e, \mu}\left(m_{b}\right)=-0.583 C_{\ell q(1)}^{e, \mu}-0.596 C_{\ell q(3)}^{e, \mu}, & C_{10}^{\mathrm{NP} e, \mu}\left(m_{b}\right)=0.588 C_{\ell q(1)}^{e, \mu}+0.591 C_{\ell q(3)}^{e, \mu}, \\
C_{V L}^{e, \mu}\left(m_{b}\right)=0.0012 C_{\ell q(1)}^{e, \mu}-0.0644 C_{\ell q(3)}^{e, \mu}, & C_{V L}^{\tau}\left(m_{b}\right)=-0.0598 C_{\ell q(3)}^{\tau} .
\end{array}
$$

It is important to note that the renormalisation group-induced SMEFT operators shift the Fermi constant $G_{F}$ [92] in the Lagrangian (9) from its SM value $G_{F}^{0}$, determined experimentally from the muon lifetime. This shift is already included in the matching conditions of Eq. (17). Note that both the contributions from the SMEFT operators entering in the redefinition of the vacuum expectation value and the ones that are relevant for the muon decay are included in our analysis. These two contributions are implemented in the package wilson [91]. The elements of the CKM matrix are also affected by SMEFT contributions [93]. Those contributions have not been included in the present work.

The $\mathcal{O}_{\ell q}$ operators (15) also produce unwanted contributions to the $B \rightarrow K^{(*)} v \bar{v}$ decays [64,94]. In order to obey these constraints, we will fix the relation at the scale $\Lambda=1 \mathrm{TeV}$

$$
C_{\ell q(1)}^{i}=C_{\ell q(3)}^{i} \equiv C_{\ell q}^{i}
$$

While the above relation eliminates the tree-level contribution to the $B \rightarrow K^{(*)} v \bar{v}$ decays, the renormalisation group (RG) generates a one-loop contribution proportional to the $C_{\ell q(3)}$ coefficients. However, we have checked that this term is only a correction of $0.1 \%$ of the SM prediction. Relation (18) also has the positive consequence of a partial cancellation of loop-induced effects in $Z$-pole and LFV observables.

Finally, an important point to emphasise here is that the dimension-six operators affect a large range of observables because of the RG equations that give mixing between different particle sectors. Therefore, any NP prediction based on Wilson coefficients has to be confronted not only with the $R_{K^{(*)}}$ an $R_{D^{(*)}}$ measurements, but also with additional several measurements involving the decays of $B$ mesons. In the case of the SMEFT, the evolution of the RG produces a mix of the low-energy effective operators. More concretely, the $\mathcal{O}_{\ell q}$ operators mix under RG evolution with [95-97]

$$
\mathcal{O}_{\varphi \ell(1)}^{j k}=\left(\varphi^{\dagger} i \overleftrightarrow{D}{ }_{\mu} \varphi\right)\left(\bar{\ell}_{j} \gamma^{\mu} \ell_{k}\right), \quad \mathcal{O}_{\varphi \ell(3)}^{j k}=\left(\varphi^{\dagger} i \overleftrightarrow{D}_{\mu}^{I} \varphi\right)\left(\bar{\ell}_{j} \gamma^{\mu} \tau^{I} \ell_{k}\right), \quad \mathcal{O}_{\varphi e}^{j k}=\left(\varphi^{\dagger} i \overleftrightarrow{D}_{\mu} \varphi\right)\left(\bar{e}_{j} \gamma^{\mu} e_{k}\right)
$$

that modify the $W$ and $Z$ couplings to leptons. In consequence, NP in the semileptonic couplings of third-generation quarks will indirectly affect electroweak observables, such as the mass of the $W$ boson, the hadronic cross section of the $Z$ boson $\sigma_{\text {had }}^{0}$ or the branching ratios of the $Z$ to different leptons. In order to keep the predictions consistent with this range of experimental test, global fits have proven to be a valuable tool $[76,77,80,81]$.

\section{Global fits}

We have performed global fits to the $C_{\ell q}$ Wilson coefficients using the package smelli v1.3 [94]. The global fit includes the $R_{K^{(*)}}$ and $R_{D^{(*)}}$ observables, the electroweak precision observables, $W$ and $Z$ decay widths and branching ratios to leptons, the $b \rightarrow s \mu \mu$ observables (including $P_{5}^{\prime}$ and the branching ratio of $B_{s} \rightarrow \mu \mu$ ) and the $b \rightarrow s v \bar{v}$ observables. The SM input parameters are presented in Table 1. These values are taken from open-source code flavio v1.5 [99]; sources used by the program are quoted when available ${ }^{2}$. Note that the experimental measurement of the $\mu \rightarrow e \bar{v} \nu$ decay, used to determine the SM input parameters, is not included in the fit in order to ensure the consistency of the procedure. The parameters $V_{u s}, V_{u b}, V_{c b}$ and $\delta_{K M}$ of the CKM matrix are treated as nuisance parameters of the fit, and the remaining elements are determined implementing the unitarity of the matrix. A complete analysis would require including the SMEFT corrections to the CKM matrix, which have not been considered in this work.

We proceed to study observables by defining some specific scenarios for combinations of the $C_{\ell q}^{i}$ operators such that NP contributions to the Wilson coefficients emerge in one, two or three of the Wilson coefficients simultaneously: in Scenarios I-III, NP only modifies the $C_{\ell q}$ operators in one lepton flavour at a time; in Scenarios IV-VI NP is present in two of the Wilson coefficients simultaneously; and finally in Scenarios VII-IX we consider the more general case in which three of the $C_{\ell q}^{i}$ operators receive NP contributions. The more general one of these last three scenarios is Scenario VII, in which we consider three independent Wilson coefficients. This scenario is discussed in more detail in Sect. 3.1.

The goodness of each fit is evaluated with its difference of $\chi^{2}$ with respect to the SM, $\Delta \chi_{\mathrm{SM}}^{2}=\chi_{\mathrm{SM}}^{2}-\chi_{\mathrm{fit}}^{2}$. The package smelli actually computes the differences of the logarithms of the likelihood function $\Delta \log L=-\frac{1}{2} \Delta \chi^{2}$. The $\chi_{\text {fit }}^{2}$ includes the experimental and theoretical uncertainties and correlations of the observables. In order to compare two fits $A$ and $B$, we use the pull between

2 We have supplemented the experimental measurements of the flavio v1.5 database with updated values for $R_{K}[15], R_{D^{(*)}}$ [21], $B \rightarrow K^{*} \ell^{+} \ell^{-}$ differential observables [104,105], $B_{(s)} \rightarrow \mu^{+} \mu^{-}$[106] and a re-analysis of the electroweak precision tests from LEP [107]. 
Table 1 SM input parameters

\begin{tabular}{lll}
\hline$G_{F}^{0}$ & $1.1663787(6) \times 10^{-5} \mathrm{GeV}^{-2}$ & PDG 2014 [98] \\
$\alpha_{e}\left(M_{Z}\right)$ & $0.00781616(86)$ & [99] \\
$\alpha_{S}\left(M_{Z}\right)$ & $0.1182(8)$ & FLAG 2019 [100] \\
$\sin ^{2} \hat{\theta}_{W}\left(M_{Z}\right), \overline{\mathrm{MS}}$ & $0.23129(5)$ & PDG 2017 [101] \\
$V_{u s}$ & $0.2248(8)$ & FLAG 2017 $N_{f}=2+1+1[102]$ \\
$\left|V_{u b}\right|$ & $3.73(14) \times 10^{-3}$ & FLAG 2017 $N_{f}=2+1 B \rightarrow \pi \ell v[102]$ \\
$V_{c b}$ & $4.221(78) \times 10^{-2}$ & {$[99]$} \\
$\delta_{\mathrm{KM}}$ & $1.27(12)$ & {$[99]$} \\
$m_{u}(2 \mathrm{GeV}), \overline{\mathrm{MS}}$ & $2.130(41) \mathrm{MeV}$ & {$[103]$} \\
$m_{d}(2 \mathrm{GeV}), \overline{\mathrm{MS}}$ & $4.675(56) \mathrm{MeV}$ & {$[103]$} \\
$m_{S}(2 \mathrm{GeV}), \overline{\mathrm{MS}}$ & $92.47(69) \mathrm{MeV}$ & {$[103]$} \\
$m_{c}\left(m_{c}\right), \overline{\mathrm{MS}}$ & $1.273(10) \mathrm{GeV}$ & {$[103]$} \\
$m_{b}\left(m_{b}\right), \overline{\mathrm{MS}}$ & $4.195(14) \mathrm{GeV}$ & {$[103]$} \\
\hline
\end{tabular}

Table 2 Best-fit values with $1 \sigma$ uncertainties and pulls from the Standard Model and of Scenario VII for several combinations of $C_{\ell q}^{i}$ operators

\begin{tabular}{|c|c|c|c|c|c|c|c|}
\hline Scenario & & $C_{\ell q}^{e}$ & $C_{\ell q}^{\mu}$ & $C_{\ell q}^{\tau}$ & $\Delta \chi_{\mathrm{SM}}^{2}$ & Pull from SM & Pull to VII \\
\hline I & $e$ & $-0.14 \pm 0.04$ & & & 8.84 & $2.97 \sigma$ & $4.37 \sigma$ \\
\hline II & $\mu$ & & $0.10 \pm 0.04$ & & 5.47 & $2.34 \sigma$ & $4.73 \sigma$ \\
\hline III & $\tau$ & & & $-0.38 \pm 0.19$ & 3.85 & $1.96 \sigma$ & $4.89 \sigma$ \\
\hline IV & $e$ and $\mu$ & $-0.25 \pm 0.07$ & $0.24 \pm 0.06$ & & 28.42 & $4.97 \sigma$ & $1.75 \sigma$ \\
\hline V & $e$ and $\tau$ & $-0.14 \pm 0.06$ & & $-0.4 \pm 0.3$ & 12.98 & $3.17 \sigma$ & $4.30 \sigma$ \\
\hline VI & $\mu$ and $\tau$ & & $0.10 \pm 0.06$ & $-0.3 \pm 0.3$ & 8.73 & $2.49 \sigma$ & $4.77 \sigma$ \\
\hline VII & $e, \mu$ and $\tau$ & $-0.25 \pm 0.02$ & $0.211 \pm 0.016$ & $-0.3 \pm 0.4$ & 31.50 & $4.97 \sigma$ & \\
\hline VIII & $e=\mu=\tau$ & $-0.0139 \pm 0.0003$ & $-0.0139 \pm 0.0003$ & $-0.0139 \pm 0.0003$ & 0.30 & $0.55 \sigma$ & $5.23 \sigma$ \\
\hline IX & $e=-\mu=\tau$ & $-0.232 \pm 0.001$ & $0.232 \pm 0.001$ & $-0.232 \pm 0.001$ & 30.74 & $5.54 \sigma$ & $0.41 \sigma$ \\
\hline
\end{tabular}

them in units of $\sigma$, defined as [108,109]

$$
\operatorname{Pull}_{A \rightarrow B}=\sqrt{2} \operatorname{Erf}^{-1}\left[F\left(\Delta \chi_{A}^{2}-\Delta \chi_{B}^{2} ; n_{B}-n_{A}\right)\right],
$$

where $\operatorname{Erf}^{-1}$ is the inverse of the error function, $F$ is the cumulative distribution function of the $\chi^{2}$ distribution and $n$ is the number of degrees of freedom of each fit. We will compare each scenario against two cases: the SM $\left(C_{\ell q}=0, n=0\right)$ and the fit to three independent Wilson coefficients (Scenario VII), which is the more general and descriptive case. The pull from the SM quantifies how much each scenario is preferred over the SM to describe the data. The larger the pull, the better the description of the data of the preferred scenario. The pull of Scenario VII quantifies how much the fit over the whole space of parameters is preferred over the simpler and more constrained fits. From the analysis of this pull, we are able to discuss the relevance of the proposed scenarios; the larger the pull means that the more restricted scenario represents a worse description of the experimental data.

The results of the fits are summarised in Table 2 for several combinations of $C_{\ell q}^{i}$ operators, with one-, two- or three-lepton flavour present simultaneously in the Wilson coefficients as defined below. The best-fit values at $1 \sigma$ and pulls from the SM and to Scenario VII for all scenarios are included in this table.

- Scenarios I, II and III: In these scenarios, NP only modifies the $C_{\ell q}^{i}$ operators in one-lepton flavour at a time, i.e. $C_{\ell q}^{e}$, $C_{\ell q}^{\mu}$ or $C_{\ell q}^{\tau}$. The largest pull from the SM prediction, almost $3 \sigma$, is found in Scenario I when the coupling to electrons is added. This result is a reflection of the great impact of the electroweak precision observables in the global fit. The fit to only muons in Scenario II displays only a pull from the SM of $2.34 \sigma$; if we restricted our fit to only $b \rightarrow s \ell^{+} \ell^{-}$observables, this fit would display a better pull, in line with the common wisdom about the anomalies, explaining them through NP in the muon sector $[37,40,75,108,110]$. The worst pull is obtained in the fit to the tau coefficient, with $1.96 \sigma$, as it does not modify the value of the $R_{K^{(*)}}$ ratios. Scenarios I and II both produce SM-like predictions for the observables $R_{D}$ and $R_{D^{*}}: R_{D}^{\ell}=0.3006$ and $R_{D^{*}}^{\ell}=0.2528$ for Scenario I and $R_{D}^{\ell}=0.3048$ and $R_{D^{*}}^{\ell}=0.2563$ for Scenario II. Scenario III, with a larger value of its Wilson coefficient, produces values closer to the average of the experimental measurements; i.e. $R_{D}^{\ell}=0.318$ and $R_{D^{*}}^{\ell}=0.268$. In order to fully address the anomaly in these observables, a larger deviation from the SM would be needed; however, such a deviation would be in conflict with the electroweak precision data, as we will see later in Sect. 3.1, and in agreement with [111]. 


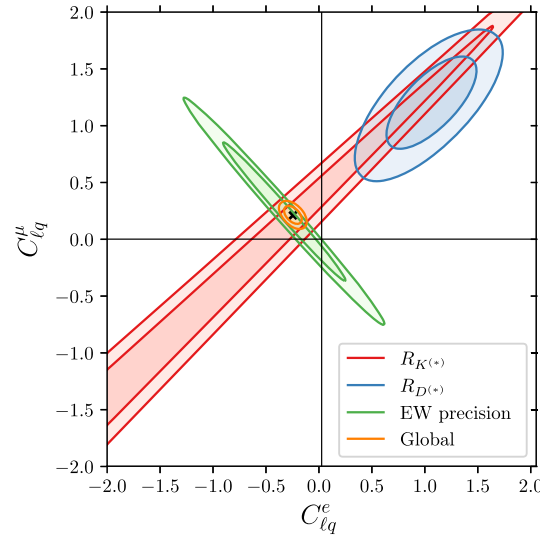

(a)

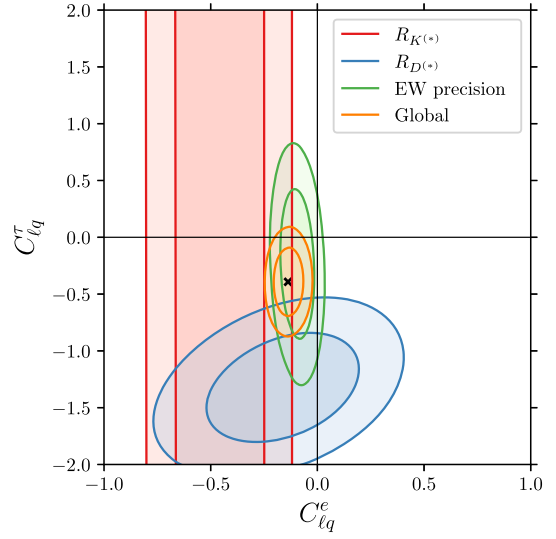

(b)

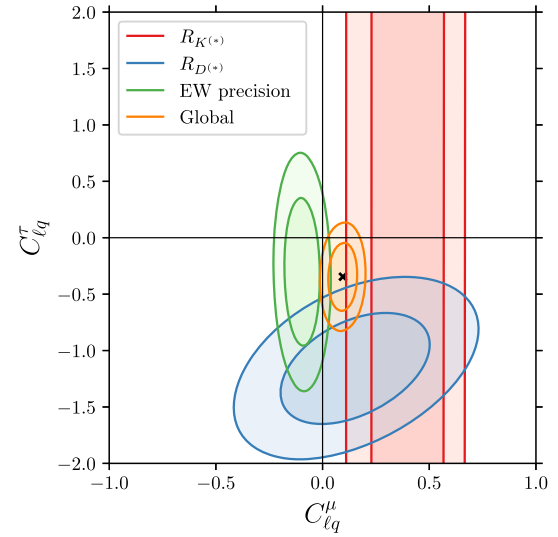

(c)

Fig. $11 \sigma$ and $2 \sigma$ contours for scenarios with two lepton flavours present in the Wilson coefficients: a Scenario IV, b Scenario V and $\mathbf{c}$ Scenario VI. All available data is considered

Table 3 Values of the $R_{K^{(*)}}$ and $R_{D^{(*)}}$ observables in the scenarios with best pulls

\begin{tabular}{llrr}
\hline Observable & Scenario IV & \multicolumn{1}{l}{ Scenario VII } & Meenario IX \\
\hline$R_{K}^{[1.1,6]}$ & $0.799 \pm 0.017$ & $0.800 \pm 0.018$ & $0.79 \pm 0.02$ \\
$R_{K^{*}}^{[0.045,1.1]}$ & $0.870 \pm 0.009$ & $0.871 \pm 0.010$ & $0.85 \pm 0.04$ \\
$R_{K^{*}}^{[1.1,6]}$ & $0.800 \pm 0.018$ & $0.802 \pm 0.019$ & $0.65 \pm 0.09$ \\
$R_{D}^{\ell}$ & $0.302 \pm 0.005$ & $0.314 \pm 0.007$ & 0.010 \\
$R_{D^{*}}^{\ell}$ & $0.254 \pm 0.004$ & $0.264 \pm 0.004$ & $0.311 \pm 0.005$ \\
$R_{D^{*}}^{\mu}$ & $0.261 \pm 0.004$ & $0.272 \pm 0.004$ & $0.261 \pm 0.004$ \\
\hline
\end{tabular}

- Scenarios IV, V and VI: In these scenarios, NP is present in two of the Wilson coefficients. The best fit corresponds to Scenario $\mathrm{IV}$, where the contributions to $C_{\ell q}^{e}$ and $C_{\ell q}^{\mu}$ are favoured with a pull of $4.97 \sigma$ with respect to the SM. Figure 1 shows the allowed regions for these fits. In the fit to Scenario IV, the $R_{K^{(*)}}$ and $R_{D^{(*)}}$ observables constrain the $C_{\ell q}^{e}-C_{\ell q}^{\mu}$ combination, while the LFU-conserving electroweak precision observables tightly constrain the combination $C_{\ell q}^{e}+C_{\ell q}^{\mu}$. It is clear that EW precision observables play an important role in the global fit and the preferred values for the Wilson coefficients. The reason for this behaviour is justified by deviations in $Z$-couplings to leptons, the $\tau$-leptonic decays and the $Z$ and $W$ decays widths, as shown in [112]. The values of the $R_{K^{(*)}}$ and $R_{D^{(*)}}$ observables in this scenario are given in Table 3 . Together, these sets of observables constrain the fit to a narrow ellipse around the best fit point. In Scenarios V and VI, the $C_{\ell q}^{\tau}$ coefficient is determined by the electroweak precision observables, that are compatible with a SM-like coefficient, and by $R_{D^{(*)}}$ observables, that prefer a large negative value. All the experimental constraints for $C_{\ell q}^{\tau}$ show large uncertainties, which result in less statistical significance of these fits and $C_{\ell q}^{\tau}$ still being compatible with zero at $2 \sigma$ level. The central values with $1 \sigma$ uncertainties of the $R_{K^{(*)}}$ and $R_{D^{(*)}}$ observables for Scenario IV (the best-fit scenario in this subset) are shown in Table 3 and Fig. 2, below we compare these results in various scenarios.

- Scenario VII: In this fit, the three $C_{\ell q}$ operators receive independent NP contribution. The pull from the SM $4.97 \sigma$, is similar to that of Scenario IV, and the values of $C_{\ell q}^{e}$ and $C_{\ell q}^{\mu}$ are similar too; therefore, the predictions for the $R_{K^{(*)}}$ observables are very similar, as shown in Fig. 2a. The value of $C_{\ell q}^{\tau}$ is close to that of Scenarios III, V and VI, which allows a better fit to the $R_{D^{(*)}}$ observables, and especially to $R_{D}^{\ell}$, that is compatible at $1 \sigma$ with its experimental value, as shown in Fig. $2 \mathrm{~b}$. Therefore, we conclude that the prediction of the $R_{D^{(*)}}$ and $R_{K^{(*)}}$ observables is improved in Scenario VII. We will discuss this scenario in more detail in Sect. 3.1.

- Scenario VIII: This scenario has universal couplings; the three Wilson coefficients have the same universal contribution and do not violate LFU. It has the smallest pull with respect to the SM $(0.30 \sigma)$. This shows that LFU NP cannot explain experimental data, and LFU violation is needed to accommodate it.

- Scenario IX: In this scenario, the three Wilson coefficients have the same absolute value, but $C_{\ell q}^{\mu}$ has the opposite sign. This particular arrangement of the coefficients was inspired by the similar absolute values of $C_{\ell q}^{e}$ and $C_{\ell q}^{\mu}$ in Scenario VII. This choice produces a good fit, with a pull of $5.54 \sigma$. It is also the only scenario that remains compatible at $1 \sigma$ with Scenario VII. 
Fig. 2 Central value and $1 \sigma$ uncertainty of the a $R_{K}(*)$ observables, and $\mathbf{b} R_{D^{(*)}}$ observables (blue lines) in Scenarios IV, VII and IX, compared to the SM prediction (yellow) and experimental measurements (green)

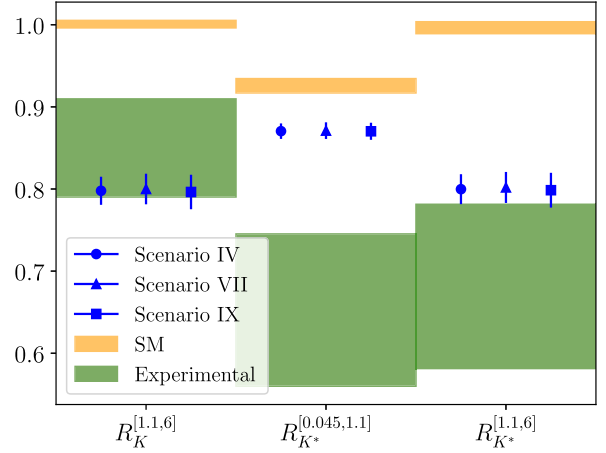

(a)

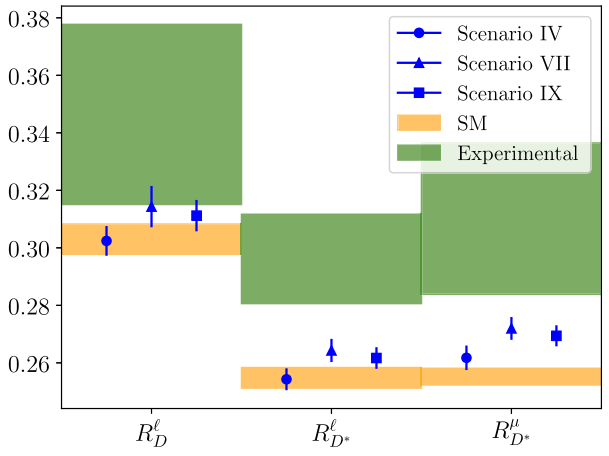

(b)

The results for the $R_{K^{(*)}}$ and $R_{D^{(*)}}$ observables in the scenarios with best pulls, Scenarios IV, VII and IX, are presented in Table 3 . Figure 2 shows the results for the central value and $1 \sigma$ uncertainty of these two observables in the three scenarios, compared to the SM prediction (yellow area) and experimental measurements (green area). These three scenarios have similar fits for the Wilson coefficients $C_{\ell q}^{e}$ and $C_{\ell q}^{\mu}$ and therefore reproduce the experimental value of $R_{K}^{[1.1,6]}$ and reduce the tension in $R_{K^{*}}^{[1.1,6]}$. The main difference between Scenarios IV, VII and IX is the fit for $C_{\ell q}^{\tau}$ : Scenario IV has no NP contribution in the $\tau$ sector and consequently predicts SM-like $R_{D^{(*)}}$ ratios; Scenario VII has a large contribution to $C_{\ell q}^{\tau}$ and is able to produce a prediction for $R_{D}^{\ell}$ compatible with the experimental results and significantly improve the predictions for $R_{D^{*}}^{\ell}$ and $R_{D^{*}}^{\mu}$; Scenario IX has an intermediate value of $C_{\ell q}^{\tau}$, and consequently its predictions for the $R_{D^{(*)}}$ ratios are not as good as in Scenario VII.

In addition to the observables included in our global fits, it is also possible to constrain the NP contributions to Wilson coefficients using high-energy collision data from LHC. In particular, it is known that high $p_{T}$ tails in proton-proton collisions producing tau leptons provide bounds that are competitive to those from the $R_{D^{(*)}}$ ratios in $B$-physics [113]. Reference [113] finds the bound $\left|C_{\ell q(3)}^{\tau}\right| / \Lambda^{2}<2.6 \mathrm{TeV}^{-2}$ by recasting the $p p \rightarrow \tau^{+} \tau^{-}$searches in ATLAS $13 \mathrm{TeV}$ with $3.2 \mathrm{fb}^{-1}$. The constraint $\left|C_{V L}^{\tau}\right|<0.32$ is established [114] for mono- $\tau$ searches $p p \rightarrow \tau X+\not_{T}$, by combining the results from ATLAS with $36.1 \mathrm{fb}^{-1}$ and CMS with $35.9 \mathrm{fb}^{-1}$, at $13 \mathrm{TeV}$. In order to compare this constraint in the WET with our fits in the SMEFT basis, we use the matching condition in Eq. (17), obtaining that $\left|C_{\ell q(3)}^{\tau}\right|<5.35$. Therefore, we can conclude that all the results of our fits are clearly compatible with the limits imposed by the high- $p_{T}$ phenomena.

\subsection{Scenario VII}

Since the Scenario VII is the more general one and we found that the prediction of the $R_{D^{(*)}}$ and $R_{K^{(*)}}$ observables is improved in this case, we discuss in this section this scenario in more detail.

The $\chi^{2}$ of the fit can be expressed as a series expansion around its minimum [109]

$$
\chi^{2}\left(C_{\ell q}^{k}\right)=\chi_{\text {fit }}^{2}+\delta C_{\ell q}^{i} \mathbb{H}_{i j} \delta C_{\ell q}^{j}+\mathcal{O}\left(\left(\delta C_{\ell q}^{k}\right)^{3}\right),
$$

where $\delta C_{\ell q}^{i}=C_{\ell q}^{i}-\left.C_{\ell q}^{i}\right|_{\mathrm{BF}}$ represent the deviation with respect to the best fit (BF) and $\mathbb{H}$ is the Hessian matrix evaluated at the best fit. In Scenario VII, the Hessian matrix takes the value:

$$
\mathbb{H}=\left(\begin{array}{ccc}
1.07524 \times 10^{4} & -1.11206 \times 10^{4} & 4.75434 \\
-1.11206 \times 10^{4} & 1.33503 \times 10^{4} & -8.39386 \\
4.75434 & -8.39386 & 26.9816
\end{array}\right)
$$

Within the quadratic approximation, the points with constant $\Delta \chi^{2}$ (e.g. all the points that are $1 \sigma$ away from the best fit) are located in the surface of an ellipsoid. The length and orientation of the ellipsoid can be found with the singular value decomposition (SVD) of the Hessian:

$$
\mathbb{H}=U \Sigma U^{T}
$$

where $U$ is an orthogonal matrix whose columns are the directions of the principal axes, and $\Sigma$ is a diagonal matrix. The lengths of the semi-axes for a given value of $\Delta \chi^{2}$ are

$$
a_{j}=\sqrt{\frac{\Delta \chi^{2}}{\Sigma_{j j}}} .
$$


In a $\chi^{2}$ distribution with three degrees of freedom, the $1 \sigma$ confidence region corresponds to $\Delta \chi^{2}=3.527$. The lengths of the semi-axes, in decreasing order, are

$$
a_{1}=0.362, \quad a_{2}=0.064, \quad a_{3}=0.0123
$$

The orientation of the axes, also in decreasing order of $a_{i}$, is given by

$$
U=\left(\begin{array}{ccc}
-0.001560 & -0.7470 & -0.6648 \\
-0.01932 & -0.6648 & 0.7470 \\
-0.9999 & -0.002450 & -4.0615 \times 10^{-4}
\end{array}\right)
$$

The first direction (i.e. the one that is less constrained by the fit) corresponds to the $\tau$ coefficient, while the second and third directions contain an equal mix of the two other Wilson coefficients that can be given as

$$
\begin{aligned}
C_{1} & \sim-C_{\ell q}^{\tau}, \\
C_{2} & \sim \frac{1}{\sqrt{2}}\left(-C_{\ell q}^{e}-C_{\ell q}^{\mu}\right), \quad C_{3} \sim \frac{1}{\sqrt{2}}\left(-C_{\ell q}^{e}+C_{\ell q}^{\mu}\right), \\
C_{\ell q}^{e} & \sim \frac{1}{\sqrt{2}}\left(-C_{2}-C_{3}\right), \quad C_{\ell q}^{\mu} \sim \frac{1}{\sqrt{2}}\left(-C_{2}+C_{3}\right) .
\end{aligned}
$$

The physical interpretation of the orientation of the axes is pretty clear from our analysis. We conclude that the NP effects in $\tau$ (axis 1) are mostly uncorrelated with those of the lighter leptons, and NP in $e$ and $\mu$ is better described as a combination of LFU effects (axis 2) and LFUV effects (axis 3). The coordinates of the best-fit point (see Scenario VII in Table 2), expressed in terms of this basis, are $C_{1}=0.336, C_{2}=0.043$ and $C_{3}=0.321$. The value obtained for the coordinate 3 implies a simultaneous decrease in the electronic part and an increase in the muonic part to describe the LFUV observables; and the value of coordinate 2 so close to 0 indicates that the LFU processes are not changed with respect to the SM.

The extrema of the $1 \sigma$ confidence ellipsoid are located at

$$
\left.C_{\ell q}^{i}\right|_{j s}=\left.C_{\ell q}^{i}\right|_{\mathrm{BF}}+s U_{i k} A_{k j}
$$

where $j=1,2,3, s= \pm 1$ and $A_{k j}=a_{j} \delta_{k j}$.

Other notable points on the ellipsoid are found moving from the best-fit point in the direction of the $C_{\ell q}^{e}, C_{\ell q}^{\mu}$ and $C_{\ell q}^{\tau}$ axes ( $j=e, \mu, \tau$ ). The distance from the best fit to the ellipsoid when changing only one Wilson coefficient $j$ is

$$
a_{j}=\sqrt{\frac{\Delta \chi^{2}}{\mathbb{H}_{j j}}}, \quad j=e, \mu, \tau,
$$

and the points of the ellipsoid obtained when only one Wilson coefficient is changed from its BF value are given by

$$
\left.C_{\ell q}^{i}\right|_{j s}=\left.C_{\ell q}^{i}\right|_{\mathrm{BF}}+s a_{j} \delta_{j}^{i}, \quad j=e, \mu, \tau
$$

Finally, the points on the $1 \sigma$ ellipsoid closest and furthest in the direction connecting the best-fit point and the SM benchmark are given by

$$
\left.C_{\ell q}^{i}\right|_{\mathrm{SM} s}=\left.C_{\ell q}^{i}\right|_{\mathrm{BF}}\left(1+s a_{\mathrm{SM}}\right)
$$

where the distance $a_{\mathrm{SM}}$ is given by

$$
a_{\mathrm{SM}}=\sqrt{\frac{\Delta \chi^{2}}{\left.\left.C_{\ell q}^{i}\right|_{\mathrm{BF}} \mathbb{H}_{i j} C_{\ell q}^{j}\right|_{\mathrm{BF}}}} .
$$

The Wilson coefficients at these points of the ellipse, from the corresponding best-fit point to the ellipsoid, at $1 \sigma$ confidence level, are given in Table 4.

The pull for a single observable is defined as

$$
\operatorname{Pull}_{\mathcal{O}}\left(C_{\ell q}\right)=\frac{\mathcal{O}\left(C_{\ell q}\right)-\mathcal{O}_{\exp }}{\sqrt{\sigma_{\exp }^{2}+\sigma_{\text {th }}^{2}\left(C_{\ell q}\right)}} .
$$


Table 4 Values of the Wilson coefficients at some points located at $1 \sigma$ confidence ellipsoid around the best-fit point in Scenario VII

\begin{tabular}{|c|c|c|c|c|c|}
\hline$j$ & $s$ & $C_{\ell q}^{e}$ & $C_{\ell q}^{\mu}$ & $C_{\ell q}^{\tau}$ & $\Delta \chi^{2}$ \\
\hline $\mathrm{BF}$ & & -0.246 & 0.211 & -0.336 & \\
\hline 1 & + & -0.246 & 0.21 & -0.698 & 3.47 \\
\hline 1 & - & -0.245 & 0.211 & 0.0251 & 3.65 \\
\hline 2 & + & -0.294 & 0.168 & -0.336 & 3.25 \\
\hline 2 & - & -0.198 & 0.253 & -0.337 & 3.22 \\
\hline 3 & + & -0.323 & 0.297 & -0.336 & 3.84 \\
\hline 3 & - & -0.168 & 0.124 & -0.336 & 3.57 \\
\hline$e$ & + & -0.159 & 0.211 & -0.336 & 3.62 \\
\hline$e$ & - & -0.332 & 0.211 & -0.336 & 3.74 \\
\hline$\mu$ & + & -0.246 & 0.292 & -0.336 & 3.71 \\
\hline$\mu$ & - & -0.246 & 0.129 & -0.336 & 3.62 \\
\hline$\tau$ & + & -0.246 & 0.211 & 0.0251 & 3.66 \\
\hline$\tau$ & - & -0.246 & 0.211 & -0.698 & 3.47 \\
\hline SM & + & -0.330 & 0.283 & -0.452 & 3.88 \\
\hline SM & - & -0.161 & 0.138 & -0.221 & 3.69 \\
\hline
\end{tabular}

Fig. 3 Pulls in the Standard Model (orange) and Scenario VII (blue) of the observables included in the global fit

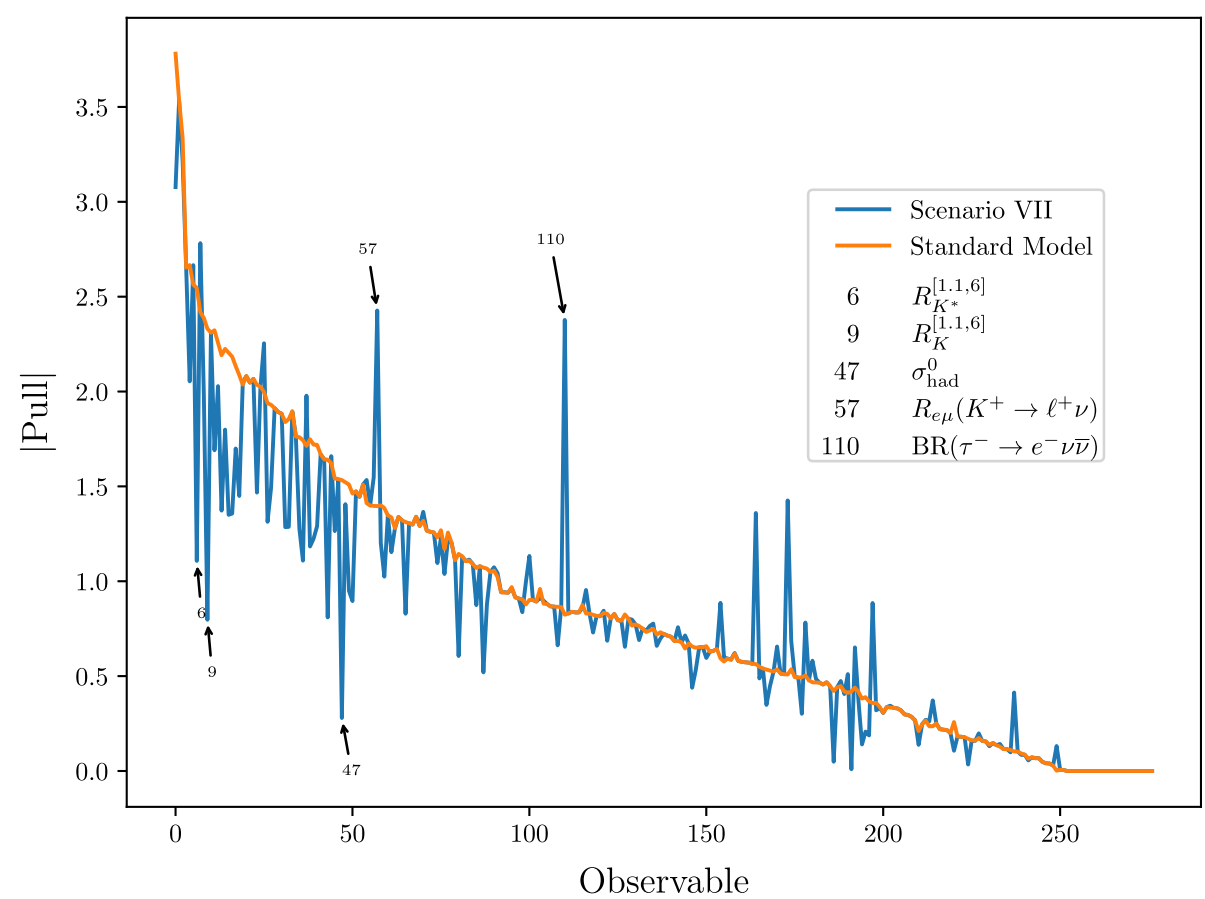

The theoretical uncertainties of the observables in general depend on the SMEFT coefficients. The package sme11i [94] treats the theoretical uncertainties in two different ways: in some observables, such as the EW precision tests, the theoretical uncertainty is considered negligible compared to the experimental uncertainty. In other cases, like the $B$-physics observables, both theoretical and experimental uncertainties are included, but they are assumed to be Gaussian. The list of observables that contribute to the global fit with their prediction in Scenario VII as well as the pulls that compare the predictions against experimental measurements for NP models (NP pull) and in the SM (SM pull) is presented in Appendix A. Notice that the values of these pulls are approximate, as they do not take in account the correlation between observables.

Figure 3 shows the pull of the observables included in the global fit for Scenario VII with respect to their experimental measurement (blue line), compared to the same pull in the SM (orange line). It is clear that, for most of the observables, the NP either improves their prediction, especially for $R_{K}, R_{K^{*}}$ (observables 9 and 6 in the table presented in Appendix A) and the hadronic $Z$ cross section 
Table 5 Observables with the largest difference of pulls between the best fit and the extreme of the $1 \sigma$ confidence ellipsoid. The number of the observables corresponds to the ones given in Appendix A

\begin{tabular}{|c|c|c|c|c|c|c|c|c|}
\hline \multicolumn{3}{|l|}{$C_{\ell q}^{e}$} & \multicolumn{3}{|l|}{$C_{\ell q}^{\mu}$} & \multicolumn{3}{|l|}{$C_{\ell q}^{\tau}$} \\
\hline No. & Observable & $\delta^{\prime 2}$ & No. & Observable & $\delta^{\prime 2}$ & No. & Observable & $\delta^{\prime 2}$ \\
\hline 39 & $m_{W}$ & 1.513 & 39 & $m_{W}$ & 1.312 & 110 & $\mathrm{BR}\left(\tau^{-} \rightarrow e^{-} v \bar{v}\right)$ & 1.060 \\
\hline 14 & $A_{e}$ & 0.418 & 9 & $R_{K}^{[1.1,6]}$ & 0.391 & 25 & $\operatorname{BR}\left(\tau^{-} \rightarrow \mu^{-} \nu \bar{v}\right)$ & 1.026 \\
\hline 9 & $R_{K}^{[1.1,6]}$ & 0.348 & 14 & $A_{e}$ & 0.290 & 47 & $\sigma_{\text {had }}^{0}$ & 0.566 \\
\hline 7 & $A_{\mathrm{FB}}$ & 0.306 & 180 & $\Gamma_{Z}$ & 0.232 & 4 & $R_{D^{*}}^{\ell}$ & 0.487 \\
\hline 180 & $\Gamma_{Z}$ & 0.268 & 7 & $A_{\mathrm{FB}}$ & 0.213 & 18 & $R_{D^{*}}^{\mu}$ & 0.179 \\
\hline \multicolumn{3}{|c|}{ Axis 1} & \multicolumn{3}{|c|}{ Axis 2} & \multicolumn{3}{|c|}{ Axis 3} \\
\hline No. & Observable & $\delta^{\prime 2}$ & No. & Observable & $\delta^{\prime 2}$ & No. & Observable & $\delta^{\prime 2}$ \\
\hline 110 & $\mathrm{BR}\left(\tau^{-} \rightarrow e^{-} \nu \bar{\nu}\right)$ & 1.055 & 39 & $m_{W}$ & 1.64 & 9 & $R_{K}^{[1.1,6]}$ & 1.419 \\
\hline 25 & $\mathrm{BR}\left(\tau^{-} \rightarrow \mu^{-} \nu \bar{v}\right)$ & 1.021 & 14 & $A_{e}$ & 0.410 & 173 & $\mathrm{BR}\left(\pi^{+} \rightarrow e \nu\right)$ & 0.475 \\
\hline 47 & $\sigma_{\text {had }}^{0}$ & 0.570 & 7 & $A_{\mathrm{FB}}$ & 0.301 & 164 & $R_{D^{*}}^{\mu / e}$ & 0.440 \\
\hline 4 & $R_{D^{*}}^{\ell}$ & 0.495 & 180 & $\Gamma_{Z}$ & 0.291 & 6 & $R_{K^{*}}^{[1.1,6]}$ & 0.276 \\
\hline 18 & $R_{D^{*}}^{\mu}$ & 0.182 & 100 & $A_{\tau}$ & 0.079 & 57 & $R_{e \mu}\left(K^{+} \rightarrow \ell^{+} \nu\right)$ & 0.135 \\
\hline \multicolumn{9}{|c|}{$\underline{\text { SM direction }}$} \\
\hline \multicolumn{3}{|l|}{ No. } & \multicolumn{3}{|c|}{ Observable } & & & $\delta^{\prime 2}$ \\
\hline \multicolumn{3}{|l|}{9} & \multicolumn{3}{|c|}{$R_{K}^{[1.1,6]}$} & & & 1.278 \\
\hline \multicolumn{3}{|l|}{173} & \multicolumn{3}{|c|}{$\mathrm{BR}\left(\pi^{+} \rightarrow e^{+} \nu\right)$} & & & 0.435 \\
\hline \multicolumn{3}{|l|}{164} & \multicolumn{3}{|c|}{$R_{D^{*}}^{\mu / e}$} & & & 0.401 \\
\hline \multicolumn{3}{|l|}{110} & \multicolumn{3}{|c|}{$\mathrm{BR}\left(\tau^{-} \rightarrow e^{-} \nu \bar{\nu}\right)$} & & & 0.287 \\
\hline \multicolumn{3}{|l|}{6} & \multicolumn{3}{|c|}{$R_{K^{*}}^{[1.1,6]}$} & & & 0.249 \\
\hline
\end{tabular}

$\sigma_{\text {had }}^{0}$ (observable 47), as well as the differential branching ratios of $B \rightarrow K^{(*)} \mu \mu$ in several low- $q^{2}$ bins; ${ }^{3}$ or leave the prediction mostly unchanged. Nevertheless, in the case of the following observables, the pull of the Scenario VII is significantly worse than that of the SM:

$$
\begin{array}{ll}
R_{e \mu}\left(K^{+} \rightarrow \ell^{+} v\right)=\frac{\mathrm{BR}\left(K^{+} \rightarrow e^{+} v\right)}{\mathrm{BR}\left(K^{+} \rightarrow \mu^{+} \nu\right)}, & \mathrm{BR}\left(\tau^{-} \rightarrow e^{-} v \bar{v}\right), \\
R_{D^{*}}^{\mu / e}=R_{\mu e}\left(B \rightarrow D^{*} \ell^{+} \nu\right)=\frac{\mathrm{BR}\left(B \rightarrow D^{*} \mu^{+} \nu\right)}{\mathrm{BR}\left(B \rightarrow D^{*} e^{+} \nu\right)}, & \mathrm{BR}\left(\pi^{+} \rightarrow e^{+} \nu\right) .
\end{array}
$$

Those observables correspond to observables 57, 110, 164 and 173, respectively, in the table given in Appendix A. Scenario VII also produces worse predictions of the $R_{K^{(*)}}$ ratios in the low-recoil bins $q^{2}>14 \mathrm{GeV}^{2}$ (observables 154 and 197 in Appendix A).

In order to identify which operators are constraining the fit in each direction, we use the difference of the pulls, defined as [109]:

$$
\delta_{j s}^{\prime}(\mathcal{O})=\operatorname{Pull}_{\mathcal{O}}\left(\left.C_{\ell q}\right|_{\mathrm{BF}}\right)-\operatorname{Pull}_{\mathcal{O}}\left(\left.C_{\ell q}\right|_{j s}\right),
$$

where $j s$ represents the direction of the corresponding axis, as described in Eqs. (29) and (31). The observables with the largest values of the square of $\delta^{\prime}$ for each extreme of the ellipse are shown in Table 5. We can see that the values of both $C_{\ell q}^{e}$ and $C_{\ell q}^{\mu}$ are constrained mostly by electroweak precision tests: the $W$-mass, the electron asymmetry in the $Z$ decay $A_{e}$, the forward-backward asymmetry $A_{\mathrm{FB}}(Z \rightarrow \bar{b} b)$ and the $Z$-decay width $\Gamma_{Z}$ (corresponding to observable 39- $m_{W}$, observable 14- $A_{e}$, observable 7- $A_{\mathrm{FB}}$ and observable $180-\Gamma_{Z}$ as presented in Appendix A), as well as by the $R_{K^{(*)}}$ data (observable 9 is $R_{K}^{[1.1,6]}$ ). The coefficient $C_{\ell q}^{\tau}$ is constrained by $\tau$ observables: the branching ratios of $\tau \rightarrow e \bar{\nu} \nu$ and $\tau \rightarrow \mu \bar{\nu} \nu$ (observables 110 and 25) and the ratios $R_{D^{*}}^{\ell}$ and $R_{D^{*}}^{\mu}$ (observables 4 and 18). This result is in agreement with [115].

${ }^{3}$ See for example observables $12,15,17,23,27,31,32,35,36,38,40,49,50,65,80,87$ in Appendix A. 


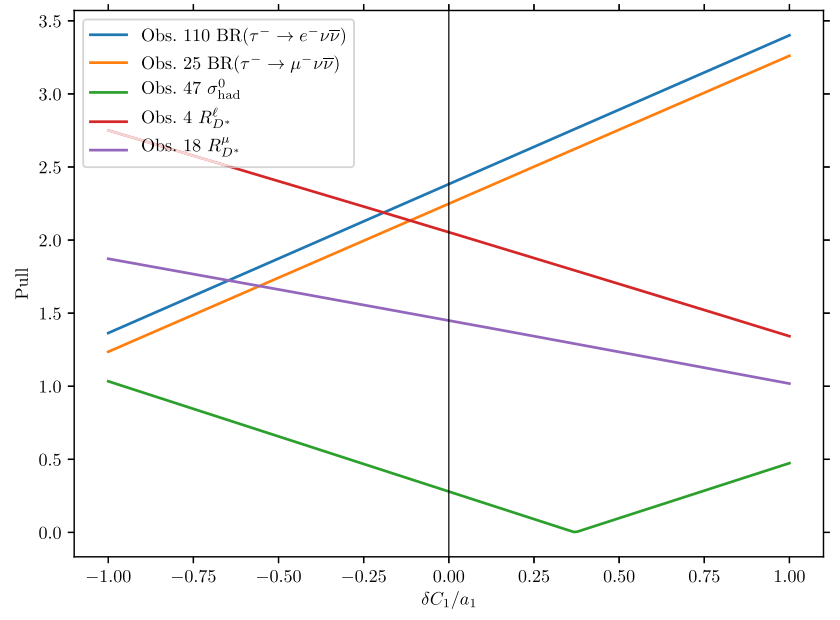

(a)

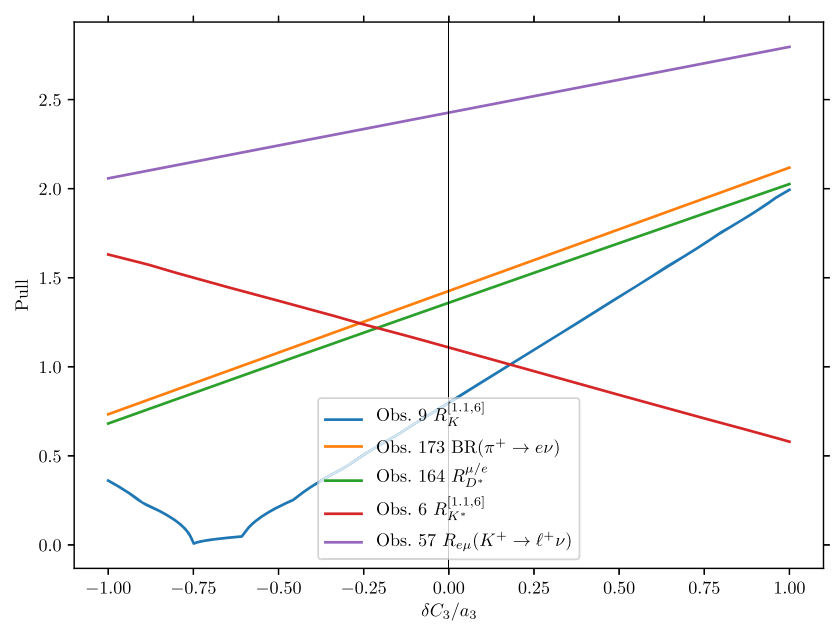

(c)

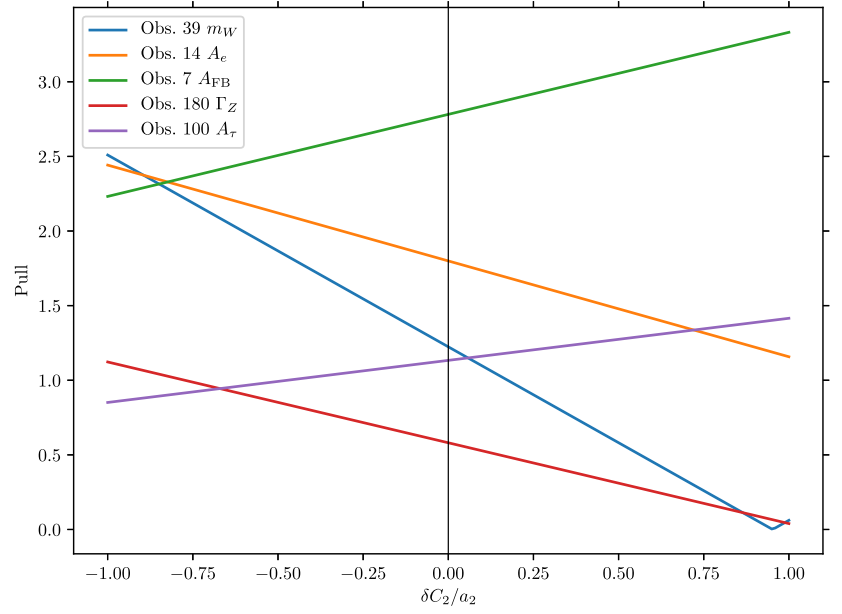

(b)

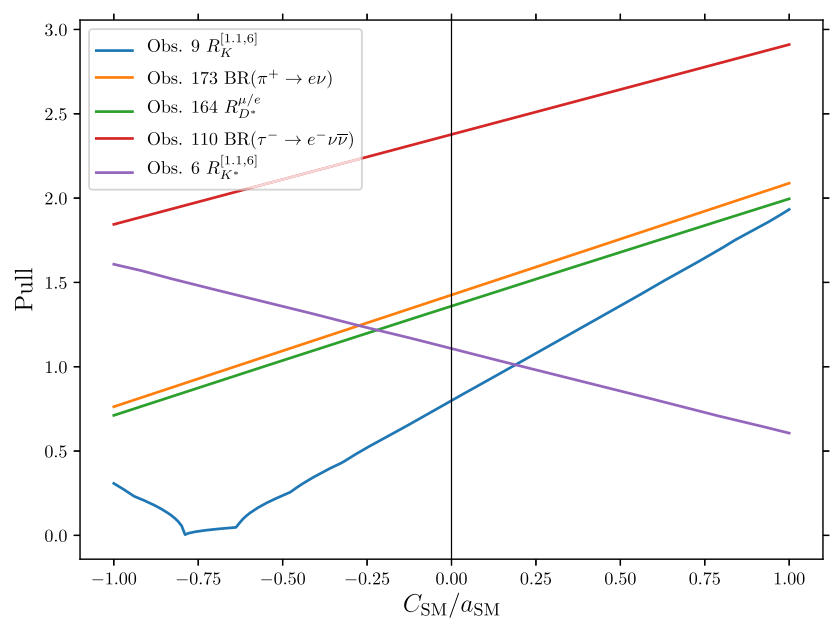

(d)

Fig. 4 Evolution of the pull of the observables in Table 5 along each axis of the ellipsoid (a)-(c) and the SM direction (d)

If we focus instead on the principal directions of the uncertainty ellipsoid, the picture is clearer: axis 1 is still dominated by $\tau$ observables. Axis 2 is constrained by the electroweak precision tests: $m_{W}, \Gamma_{Z}, A_{\mathrm{FB}}(Z \rightarrow \bar{b} b)$ and the leptonic asymmetries $A_{e}$ and $A_{\tau}$ (observables 14 and 100). Axis 3 , on the other hand, is constrained by observables sensitive to lepton universality violations in the $e$ - $\mu$ sector: $R_{K^{(*)}}$ observables (observable 9 is $R_{K}^{[1,1,6]}$ and observable 6 is $R_{K^{*}}^{[1.1,6]}$ ), but also the equivalent $R_{D^{(*)}}$ observable $R_{\mu e}\left(B \rightarrow D^{*} \ell^{+} v\right)$ (observable 164), the leptonic branching ratio of $\pi^{+} \rightarrow e^{+} v$ (observable 173) and the ratio $R_{e \mu}\left(K^{+} \rightarrow \ell^{+} v\right)$ (observable 78), all of them defined in (35). Indeed, this separation between electroweak and $R_{K^{(*)}}$ observables is already visible in Figure 1a: the allowed region by EW precision observables (green) is focused around a constant value of $C_{\ell q}^{e}+C_{\ell q}^{\mu}$ approximately aligned with axis 3 , while the allowed region of the $R_{K^{(*)}}$ observables (red) is focused around a constant value of $C_{\ell q}^{e}-C_{\ell q}^{\mu}$, approximately aligned with axis 2 .

Figure 4 represents the evolution of these observables along the axes of the ellipsoid (see Eq. (27) for definitions of $C_{1}, C_{2}, C_{3}$ ). In the case of the first axis, $\delta C_{1} / a_{1}=-1$ corresponds to a suppression of NP in the $\tau$ sector, which is preferred by the $\tau$ decays, while $\delta C_{1} / a_{1}=1$ is an increase in $\tau$ effects with respect to the best fit, that accommodates better the $R_{D^{(*)}}$ anomalies, as was previously pointed out in [111]. In the second axis, the observables $A_{\mathrm{FB}}$ and $A_{\tau}$ favour a decrease in the flavour universal NP contribution, while $A_{e}, m_{W}$ and $\Gamma_{Z}$ prefer lower contributions, with the two latter observables attaining their experimental values. In the case of axis $3, \delta C_{3} / a_{3}=1$ favours NP effects in muons (it increases $C_{\ell q}^{\mu} \sim-C_{9}$, and a deficit of muons needs a negative $C_{9}$ ) while $\delta C_{3} / a_{3}=-1$ favours NP effects in electrons: $R_{K}$ prefers a smaller contribution to the muonic part, while $R_{K^{*}}$ prefers a larger contribution. This is consistent with Fig. 2, where the prediction for $R_{K}$ is below its central experimental value and the prediction of $R_{K^{*}}$ is above its experimental value. The other LFUV observables also prefer smaller muonic NP effects.

The last columns of Table 5 and Fig. 4d show the observables that constrain the fit along the direction connecting the SM and best-fit point, that is in the points with Wilson coefficients of the form $C_{\ell q}^{i}=\left.C_{\ell q}^{i}\right|_{\mathrm{BF}}\left(1+\delta C_{\mathrm{SM}}\right)$. We observe that this direction is 
determined mostly by the LFUV observables $R_{K}^{[1.1,6]}, R_{K^{*}}^{[1.1,6]}, R_{D^{*}}^{\mu / e}$, the $\tau$ decay $\mathrm{BR}\left(\tau^{-} \rightarrow e^{-} \nu \bar{\nu}\right)$ and $\mathrm{BR}\left(\pi^{+} \rightarrow e^{+} \nu\right)$. These are the observables whose pulls change the most when comparing the best fit and SM, and therefore the ones more relevant to constrain the fit. In particular, the fit is optimal for $R_{K}^{[1.1,6]}$, a larger deviation would be needed for $R_{K^{*}}^{[1.1,6]}$, while $R_{D^{*}}^{\mu / e}, \mathrm{BR}\left(\tau^{-} \rightarrow e^{-} \nu \bar{\nu}\right)$ and $\mathrm{BR}\left(\pi^{+} \rightarrow e^{+} \nu\right)$ would be better explained with a SM-like arrangement.

\section{Connection to leptoquark models}

For completeness, we discuss in this section the phenomenological implications of our assumptions in the leptoquark models, concretely in the vector leptoquark model. The vector leptoquark $U_{1}=(\overline{\mathbf{3}}, \mathbf{1})_{2 / 3}$ couples to left-handed and right-handed fermions as

$$
\mathcal{L}=x_{L}^{i j} \bar{q}_{i} \gamma_{\mu} U_{1}^{\mu} \ell_{j}+x_{R}^{i j} \bar{d}_{R i} \gamma_{\mu} U_{1}^{\mu} e_{R j}+\text { h.c. }
$$

where $d_{R}$ and $e_{R}$ are the $d$-type quark and charged lepton $S U(2)$ singlets, and $x_{L}$ and $x_{R}$ are the matrices of couplings of the $U_{1}$ leptoquark to left-handed and right-handed fermions, respectively.

When matched with the SMEFT at the scale $\Lambda$, an $U_{1}$ leptoquark with mass $M_{U}$ contributes to the following Wilson coefficients [116]:

$$
\begin{gathered}
C_{\ell q(1)}^{i j k l}=C_{\ell q(3)}^{i j k l}=\frac{-\Lambda^{2}}{2 M_{U}^{2}} x_{L}^{l i} x_{L}^{k j *}, \\
C_{e d}^{i j k l}=-\frac{1}{2} C_{l e d q}^{i j k l}=\frac{-\Lambda^{2}}{M_{U}^{2}} x_{L}^{l i} x_{R}^{k j *} .
\end{gathered}
$$

If we only allow couplings to the left-handed fermions, the leptoquark only affects $C_{\ell q}$, as we used in our assumptions. The coefficients used in Scenarios I through IX in terms of the leptoquarks couplings are

$$
C_{\ell q}^{e}=-\frac{\Lambda^{2}}{2 M_{U}^{2}}\left|x_{L}^{b e}\right|^{2} \quad C_{\ell q}^{\mu}=-\frac{\Lambda^{2}}{2 M_{U}^{2}}\left|x_{L}^{b \mu}\right|^{2} \quad C_{\ell q}^{\tau}=-\frac{\Lambda^{2}}{2 M_{U}^{2}}\left|x_{L}^{b \tau}\right|^{2},
$$

which obviously must be negative real numbers.

According to the results of the fits in Table 2, the scenarios that include NP contributions in the electronic or tau sectors show preference for negative values of $C_{\ell q}^{e}$ and $C_{\ell q}^{\tau}$ and thus can be described by a $U_{1}$ leptoquark. On the contrary, all the fits to scenarios affecting the muon coupling show clear preference for positive values of the Wilson coefficient $C_{\ell q}^{\mu}$. In consequence, with our assumptions, the leptoquark $U_{1}$ cannot describe the anomalies in the muon sector and, therefore, does not play an important role in describing the LFUV, as shown by the fact that the scenarios with a greater pull from the SM, Scenarios IV, VII and IX, are not compatible. These results confirm previous results which have shown that the $U_{1}$ leptoquark models cannot describe the anomalies on $R_{K^{(*)}}$ and can only address the deficit in this observable when it has both couplings to $b \mu$ and $s \mu$ (see, for example [29]).

Other leptoquark models do not retain the $C_{\ell q(1)}=C_{\ell q(3)}$ condition [61,116] and therefore produce large contributions to the $B \rightarrow K^{(*)} \nu \bar{v}$ decays. That is the case of the scalar $S_{3}=(\overline{\mathbf{3}}, \mathbf{3})_{1 / 3}$, that predicts $C_{\ell q(1)}=3 C_{\ell q(3)}$, and the vector $U_{3}=(\overline{\mathbf{3}}, \mathbf{3})_{2 / 3}$, where $C_{\ell q(1)}=-3 C_{\ell q(3)}$. The scalar $S_{1}=(\overline{\mathbf{3}}, \mathbf{1})_{1 / 3}$ is even less suited, as it predicts $C_{\ell q(1)}=-C_{\ell q(3)}$, which would result in no NP contributing to $b \rightarrow s \ell^{+} \ell^{-}$at all. New vector bosons $W^{\prime}$ and $Z^{\prime}$ would also be in conflict with the $B \rightarrow K^{(*)} \nu \bar{v}$ decays, as they predict $C_{\ell q(1)}=0$ while $C_{\ell q(3)}$ has a nonzero value.

\section{Conclusions}

Several measurements of $B$ meson decays performed in the recent years indicate a possible violation of lepton universality that may represent an indirect signal of New Physics. In this work, we provide an analysis of the effects of the global fits to the Wilson coefficients assuming a model-independent effective Hamiltonian approach and including a discussion of the consequences of our assumptions on the analysis in leptoquark models. The global fit includes $b \rightarrow s \mu \mu$ observables (including the lepton flavour universality ratios $R_{K^{(*)}}$, the angular observables $P_{5}^{\prime}$ and the branching ratio of $B_{s} \rightarrow \mu \mu$ ), as well as the $R_{D^{(*)}}, b \rightarrow s \nu \bar{v}$ and electroweak precision observables ( $W$ and $Z$ decay widths and branching ratios to leptons).

We consider different scenarios for the phenomenological analysis such that New Physics is present in one, two or three of the Wilson coefficients at a time (Table 2), with the choice of the effective operators motivated by a $U(2)^{3}$ symmetry between light quarks. Our results are relevant for model-independent analysis, clarifying which combinations of the Wilson coefficients are constrained by the data. For all scenarios, we compare the results of the global fit with respect to both the SM and the more general and descriptive scenario: the best-fit point of the three independent Wilson coefficients scenario in which New Physics modifies each of the operators independently. 
We conclude that, when New Physics contributes to only one lepton flavour operator at a time, the largest pull from the Standard Model prediction, almost $3 \sigma$ (Table 2), appears when the coupling to electrons is added independently, corresponding to our Scenario I. In those scenarios in which New Physics is present in two of the Wilson coefficients simultaneously, the best fit corresponds to the case of Scenario IV, where the contributions to $C_{\ell q}^{e}$ and $C_{\ell q}^{\mu}$ are favoured with a pull of $4.97 \sigma$ with respect to the SM (Table 2). In this case, we confirm that the $R_{K^{(*)}}$ and $R_{D^{(*)}}$ constrain the linear combination $C_{\ell q}^{e}-C_{\ell q}^{\mu}$, while the LFU-conserving electroweak precision observables constrain $C_{\ell q}^{e}+C_{\ell q}^{\mu}$.

If we focus on the more general and descriptive scenario of three independent Wilson coefficients, we found that the prediction of the $R_{D^{(*)}}$ and $R_{K^{(*)}}$ observables is improved in the scenario in which the three $C_{\ell q}$ operators receive independent NP contributions: Scenario VII. In this case, the pull from the Standard Model is $4.97 \sigma$ (Table 2) and the predictions for the $R_{K^{(*)}}$ observables are very similar to the case of Scenario IV. A better fit to $R_{D^{(*)}}$ observables, and specially to $R_{D}^{\ell}$, is obtained in this scenario. We have also analysed which observables constrain the fit in each direction using the difference of their pulls: the values of both $C_{\ell q}^{e}$ and $C_{\ell q}^{\mu}$ are constrained mostly by electroweak precision tests. A clear separation between electroweak and LFU observables is established, with electroweak precision observables focused around a constant value of $C_{\ell q}^{e}+C_{\ell q}^{\mu}$, while the allowed region of the $R_{K^{(*)}}$ observables is focused around a constant value of $C_{\ell q}^{e}-C_{\ell q}^{\mu}$ requiring a large violation of lepton flavour universality. From our analysis, we also conclude that the more relevant observables in the global fit are the LFUV observables $R_{K}^{[1.1,6]}, R_{K^{*}}^{[1.1,6]}, R_{D^{*}}^{\mu / e}$ and the branching ratio of the $\tau$ decay $\mathrm{BR}\left(\tau^{-} \rightarrow e^{-} \nu \bar{\nu}\right)$, given that these observables exhibit the larger change in their pulls along the direction connecting the SM and best fit point, that is $C_{\ell q}^{i}=\left.C_{\ell q}^{i}\right|_{\mathrm{BF}}\left(1+\delta C_{\mathrm{SM}}\right)$.

Scenario IX (Table 2) represents a much more restricted scenario with only one free Wilson coefficient; nevertheless, it provides a good fit to experimental data, with a pull of $5.55 \sigma$ with respect to the SM, and it is compatible with Scenario VII at $0.41 \sigma$; therefore, it provides a similar description to experimental data with less free parameters.

Summarising, Scenario VII (three independent Wilson coefficients) is the favoured one for explaining the tension between SM predictions and $B$-physics anomalies, with Scenario IX providing a similar fit goodness with a smaller set of free parameters.

Finally, we compare our setting to the $U_{1}$ leptoquark model. We conclude that, with our assumptions, this model cannot describe the anomalies in the muon sector and, therefore, does not play an important role in describing the LFUV. Other leptoquark models do not contribute to the effective operators that we consider in this work.

Acknowledgements The work of J. A. and S. P. is partially supported by Spanish grants MINECO/FEDER, grant FPA2015-65745-P, PGC2018-095328-BI00 (FEDER/Agencia estatal de investigación) and DGIID-DGA No. 2015-E24/2. J. A. is also supported by the Departamento de Innovación, Investigación y Universidad of Aragón government, Grant No. DIIU-DGA. J.G. has been supported by MICIN under projects PID2019-105614GB-C22 and CEX2019000918-M of ICCUB (Unit of Excellence María de Maeztu 2020-2023) and AGAUR (2017SGR754).

Funding Open Access funding provided thanks to the CRUE-CSIC agreement with Springer Nature.

Open Access This article is licensed under a Creative Commons Attribution 4.0 International License, which permits use, sharing, adaptation, distribution and reproduction in any medium or format, as long as you give appropriate credit to the original author(s) and the source, provide a link to the Creative Commons licence, and indicate if changes were made. The images or other third party material in this article are included in the article's Creative Commons licence, unless indicated otherwise in a credit line to the material. If material is not included in the article's Creative Commons licence and your intended use is not permitted by statutory regulation or exceeds the permitted use, you will need to obtain permission directly from the copyright holder. To view a copy of this licence, visit http://creativecommons.org/licenses/by/4.0/.

\section{A Pulls of the observables in Scenario VII}

This table contains all observables that contribute to the global fit, as well as their prediction in Scenario VII and their pull in both Scenario VII (NP pull) and SM (SM pull). Predictions for dimensionful observables are expressed in the corresponding power of $\mathrm{GeV}$ (for example, $\Delta M_{s}$ in $\mathrm{GeV}$ and $\sigma_{\text {had }}^{0}$ in $\mathrm{GeV}^{-2}$ ). The notation $\langle\cdots\rangle$ means that the observable is binned in the invariant mass-squared of the di-lepton system $q^{2}$, with the endpoints of the bin in $\mathrm{GeV}^{2}$ given in the superscript. Accordingly, the notation $\frac{\langle\mathrm{BR}\rangle}{\mathrm{BR}}$ denotes a binned branching ratio normalised to the total branching ratio. Observables are ordered according to their SM pull and color-coded according to the difference between the Scenario VII and SM pulls: green observables have a better pull in Scenario VII, red observables have a better pull in the SM, and white observables have a similar pull in both cases.

Notice that not all observables are affected by NP in our scenario. However, the inclusion of these observables does not alter the value of the $\Delta \chi^{2}$, since their prediction and uncertainty are unchanged from the SM, and the statistical significance of the fit remains unchanged. 


\begin{tabular}{|c|c|c|c|c|}
\hline & Observable & NP prediction & NP pull & SM pull \\
\hline 0 & $\left\langle\frac{d \overline{\mathrm{BR}}}{d q^{2}}\right\rangle\left(B_{s} \rightarrow \phi \mu^{+} \mu^{-}\right)^{[1.0,6.0]}$ & $4.864 \times 10^{-8}$ & $3.1 \sigma$ & $3.8 \sigma$ \\
\hline 1 & $a_{\mu}$ & 0.0011659 & $3.5 \sigma$ & $3.5 \sigma$ \\
\hline 2 & $\left\langle P_{5}^{\prime}\right\rangle\left(B^{0} \rightarrow K^{* 0} \mu^{+} \mu^{-}\right)^{[4,6]}$ & -0.73724 & $3.2 \sigma$ & $3.3 \sigma$ \\
\hline 3 & $\epsilon^{\prime} / \epsilon$ & $-2.9466 \times 10^{-5}$ & $2.7 \sigma$ & $2.7 \sigma$ \\
\hline 4 & $R_{\tau \ell}\left(B \rightarrow D^{*} \ell^{+} \nu\right)$ & 0.26431 & $2.1 \sigma$ & $2.7 \sigma$ \\
\hline 5 & $\mathrm{BR}\left(W^{ \pm} \rightarrow \tau^{ \pm} \nu\right)$ & 0.1082 & $2.7 \sigma$ & $2.6 \sigma$ \\
\hline 6 & $\left\langle R_{\mu e}\right\rangle\left(B^{0} \rightarrow K^{* 0} \ell^{+} \ell^{-}\right)^{[1.1,6.0]}$ & 0.80189 & $1.1 \sigma$ & $2.5 \sigma$ \\
\hline 7 & $A_{\mathrm{FB}}^{0, b}$ & 0.10365 & $2.8 \sigma$ & $2.4 \sigma$ \\
\hline 8 & $\left\langle R_{\mu e}\right\rangle\left(B^{0} \rightarrow K^{* 0} \ell^{+} \ell^{-}\right)^{[0.045,1.1]}$ & 0.87107 & $2 \sigma$ & $2.4 \sigma$ \\
\hline 9 & $\left\langle R_{\mu e}\right\rangle\left(B^{ \pm} \rightarrow K^{ \pm} \ell^{+} \ell^{-}\right)^{[1.1,6.0]}$ & 0.8 & $0.8 \sigma$ & $2.3 \sigma$ \\
\hline 10 & $\frac{\langle\mathrm{BR}\rangle}{\mathrm{BR}}\left(B \rightarrow D^{*} \tau^{+} \nu\right)^{[10.4,10.93]}$ & 0.019509 & $2.3 \sigma$ & $2.3 \sigma$ \\
\hline 11 & $\left\langle\frac{d \mathrm{BR}}{d q^{2}}\right\rangle\left(B^{+} \rightarrow K^{*+} \mu^{+} \mu^{-}\right)^{[15.0,19.0]}$ & $5.7706 \times 10^{-8}$ & $1.7 \sigma$ & $2.3 \sigma$ \\
\hline 12 & $\left\langle P_{2}\right\rangle\left(B^{0} \rightarrow K^{* 0} \mu^{+} \mu^{-}\right)^{[4,6]}$ & 0.26583 & $2 \sigma$ & $2.3 \sigma$ \\
\hline 13 & $\overline{\mathrm{BR}}\left(B_{s} \rightarrow \mu^{+} \mu^{-}\right)$ & $3.31 \times 10^{-9}$ & $1.4 \sigma$ & $2.2 \sigma$ \\
\hline 14 & $A_{e}$ & 0.14785 & $1.8 \sigma$ & $2.2 \sigma$ \\
\hline 15 & $\left\langle\frac{d \mathrm{BR}}{d q^{2}}\right\rangle\left(B^{0} \rightarrow K^{* 0} \mu^{+} \mu^{-}\right)^{[15.0,19.0]}$ & $5.3257 \times 10^{-8}$ & $1.4 \sigma$ & $2.2 \sigma$ \\
\hline 16 & $\left\langle\frac{d \overline{\mathrm{BR}}}{d q^{2}}\right\rangle\left(B_{s} \rightarrow \phi \mu^{+} \mu^{-}\right)^{[15.0,19.0]}$ & $4.9957 \times 10^{-8}$ & $1.4 \sigma$ & $2.2 \sigma$ \\
\hline 17 & $\left\langle\frac{d \mathrm{BR}}{d q^{2}}\right\rangle\left(B^{+} \rightarrow K^{*+} \mu^{+} \mu^{-}\right)^{[4.0,6.0]}$ & $4.8554 \times 10^{-8}$ & $1.7 \sigma$ & $2.1 \sigma$ \\
\hline 18 & $R_{\tau \mu}\left(B \rightarrow D^{*} \ell^{+} \nu\right)$ & 0.27198 & $1.4 \sigma$ & $2.1 \sigma$ \\
\hline 19 & $\left\langle A_{\mathrm{FB}}^{\ell h}\right\rangle\left(\Lambda_{b} \rightarrow \Lambda \mu^{+} \mu^{-}\right)^{[15,20]}$ & 0.16297 & $2 \sigma$ & $2 \sigma$ \\
\hline 20 & $\left|\epsilon_{K}\right|$ & 0.0018127 & $2.1 \sigma$ & $2.1 \sigma$ \\
\hline 21 & $\operatorname{BR}\left(B^{ \pm} \rightarrow K^{ \pm} \tau^{+} \tau^{-}\right)$ & $1.9363 \times 10^{-7}$ & $2 \sigma$ & $2 \sigma$ \\
\hline 22 & $\mathrm{BR}\left(K_{L} \rightarrow e^{+} e^{-}\right)$ & $1.9327 \times 10^{-13}$ & $2.1 \sigma$ & $2.1 \sigma$ \\
\hline 23 & $\left\langle\frac{d \mathrm{BR}}{d q^{2}}\right\rangle\left(B^{ \pm} \rightarrow K^{ \pm} \mu^{+} \mu^{-}\right)^{[4.0,5.0]}$ & $3.1212 \times 10^{-8}$ & $1.5 \sigma$ & $2 \sigma$ \\
\hline 24 & $\frac{\langle\mathrm{BR}\rangle}{\mathrm{BR}}\left(B \rightarrow D^{*} \tau^{+} \nu\right)^{[5.07,5.6]}$ & 0.059848 & $2 \sigma$ & $2 \sigma$ \\
\hline 25 & $\mathrm{BR}\left(\tau^{-} \rightarrow \mu^{-} \nu \bar{\nu}\right)$ & 0.17277 & $2.3 \sigma$ & $2 \sigma$ \\
\hline 26 & $\left\langle\frac{d \mathrm{BR}}{d q^{2}}\right\rangle\left(B^{0} \rightarrow K^{0} \mu^{+} \mu^{-}\right)^{[15.0,22.0]}$ & $1.2492 \times 10^{-8}$ & $1.3 \sigma$ & $1.9 \sigma$ \\
\hline 27 & $\left\langle\frac{d \mathrm{BR}}{d q^{2}}\right\rangle\left(B^{0} \rightarrow K^{0} \mu^{+} \mu^{-}\right)^{[4.0,6.0]}$ & $2.8846 \times 10^{-8}$ & $1.5 \sigma$ & $1.9 \sigma$ \\
\hline 28 & $a_{e}$ & 0.0011597 & $1.9 \sigma$ & $1.9 \sigma$ \\
\hline 29 & $\frac{\langle\mathrm{BR}\rangle}{\mathrm{BR}}\left(B \rightarrow D \tau^{+} \nu\right)^{[7.73,8.27]}$ & 0.091906 & $1.9 \sigma$ & $1.9 \sigma$ \\
\hline 30 & $\frac{\langle\mathrm{BR}\rangle}{\mathrm{BR}}\left(B \rightarrow D^{*} \tau^{+} \nu\right)^{[7.2,7.73]}$ & 0.10205 & $1.9 \sigma$ & $1.9 \sigma$ \\
\hline 31 & $\left\langle\frac{d \mathrm{BR}}{d q^{2}}\right\rangle\left(B^{ \pm} \rightarrow K^{ \pm} \mu^{+} \mu^{-}\right)^{[1.1,2.0]}$ & $3.1704 \times 10^{-8}$ & $1.3 \sigma$ & $1.8 \sigma$ \\
\hline 32 & $\left\langle\frac{d \mathrm{BR}}{d q^{2}}\right\rangle\left(B^{ \pm} \rightarrow K^{ \pm} \mu^{+} \mu^{-}\right)^{[5.0,6.0]}$ & $3.0985 \times 10^{-8}$ & $1.3 \sigma$ & $1.9 \sigma$ \\
\hline 33 & $\left\langle P_{1}\right\rangle\left(B^{0} \rightarrow K^{* 0} \mu^{+} \mu^{-}\right)^{[4.3,6]}$ & -0.17908 & $1.9 \sigma$ & $1.9 \sigma$ \\
\hline 34 & $F_{L}\left(B^{0} \rightarrow D^{*-} \tau^{+} \nu_{\tau}\right)$ & 0.44235 & $1.8 \sigma$ & $1.8 \sigma$ \\
\hline 35 & $\left\langle\frac{d \mathrm{BR}}{d q^{2}}\right\rangle\left(B^{0} \rightarrow K^{* 0} \mu^{+} \mu^{-}\right)^{[1.1,2.5]}$ & $4.2757 \times 10^{-8}$ & $1.3 \sigma$ & $1.8 \sigma$ \\
\hline 36 & $\left\langle\frac{d \mathrm{BR}}{d q^{2}}\right\rangle\left(B^{0} \rightarrow K^{* 0} \mu^{+} \mu^{-}\right)^{[4.0,6.0]}$ & $4.4839 \times 10^{-8}$ & $1.1 \sigma$ & $1.7 \sigma$ \\
\hline 37 & $\left\langle\frac{d \mathrm{BR}}{d q^{2}}\right\rangle\left(\Lambda_{b} \rightarrow \Lambda \mu^{+} \mu^{-}\right)^{[15,20]}$ & $6.3747 \times 10^{-8}$ & $2 \sigma$ & $1.7 \sigma$ \\
\hline 38 & $\left\langle\frac{d \mathrm{BR}}{d q^{2}}\right\rangle\left(B^{0} \rightarrow K^{* 0} \mu^{+} \mu^{-}\right)^{[4.3,6]}$ & $4.5307 \times 10^{-8}$ & $1.2 \sigma$ & $1.7 \sigma$ \\
\hline 39 & $m_{W}$ & 80.365 & $1.2 \sigma$ & $1.7 \sigma$ \\
\hline
\end{tabular}




\begin{tabular}{|c|c|c|c|c|}
\hline & Observable & NP prediction & NP pull & SM pull \\
\hline 40 & $\left\langle\frac{d \mathrm{BR}}{d q^{2}}\right\rangle\left(B^{0} \rightarrow K^{0} \mu^{+} \mu^{-}\right)^{[2.0,4.0]}$ & $2.9205 \times 10^{-8}$ & $1.3 \sigma$ & $1.7 \sigma$ \\
\hline 41 & $A_{\Delta \Gamma}\left(B_{s} \rightarrow \phi \gamma\right)$ & 0.030556 & $1.7 \sigma$ & $1.7 \sigma$ \\
\hline 42 & $\frac{\langle\mathrm{BR}\rangle}{\mathrm{BR}}\left(B \rightarrow D \tau^{+} \nu\right)^{[9.0,9.5]}$ & 0.068292 & $1.6 \sigma$ & $1.6 \sigma$ \\
\hline 43 & $\left\langle\frac{d \mathrm{BR}}{d q^{2}}\right\rangle\left(B^{ \pm} \rightarrow K^{ \pm} \mu^{+} \mu^{-}\right)^{[15.0,22.0]}$ & $1.3561 \times 10^{-8}$ & $0.81 \sigma$ & $1.6 \sigma$ \\
\hline 44 & $\left\langle F_{L}\right\rangle\left(B^{0} \rightarrow K^{* 0} \mu^{+} \mu^{-}\right)^{[4,6]}$ & 0.71389 & $1.7 \sigma$ & $1.6 \sigma$ \\
\hline 45 & $R_{\mu}^{0}$ & 20.743 & $1.3 \sigma$ & $1.5 \sigma$ \\
\hline 46 & $\left\langle D_{P_{5}^{\prime}}^{\mu e}\right\rangle\left(B^{0} \rightarrow K^{* 0} \ell^{+} \ell^{-}\right)^{[14.18,19.0]}$ & 0.0027745 & $1.5 \sigma$ & $1.5 \sigma$ \\
\hline 47 & $\sigma_{\text {had }}^{0}$ & 0.00010666 & $0.28 \sigma$ & $1.5 \sigma$ \\
\hline 48 & $A_{\mathrm{FB}}^{0, \tau}$ & 0.016409 & $1.4 \sigma$ & $1.5 \sigma$ \\
\hline 49 & $\left\langle\frac{d \mathrm{BR}}{d q^{2}}\right\rangle\left(B^{ \pm} \rightarrow K^{ \pm} \mu^{+} \mu^{-}\right)^{[3.0,4.0]}$ & $3.1401 \times 10^{-8}$ & $0.95 \sigma$ & $1.5 \sigma$ \\
\hline 50 & $\left\langle\frac{d \mathrm{BR}}{d q^{2}}\right\rangle\left(B^{0} \rightarrow K^{* 0} \mu^{+} \mu^{-}\right)^{[2.5,4.0]}$ & $4.0397 \times 10^{-8}$ & $0.9 \sigma$ & $1.5 \sigma$ \\
\hline 51 & $\mathrm{BR}\left(B^{-} \rightarrow \pi^{-} e^{+} \tau^{-}\right)$ & 0 & $1.5 \sigma$ & $1.5 \sigma$ \\
\hline 52 & $\mathrm{BR}\left(B^{+} \rightarrow K^{+} \nu \bar{\nu}\right)$ & $4.3974 \times 10^{-6}$ & $1.4 \sigma$ & $1.4 \sigma$ \\
\hline 53 & $\left\langle\overline{S_{4}}\right\rangle\left(B_{s} \rightarrow \phi \mu^{+} \mu^{-}\right)^{[15.0,19.0]}$ & -0.3018 & $1.5 \sigma$ & $1.5 \sigma$ \\
\hline 54 & $\mathrm{BR}\left(W^{ \pm} \rightarrow \mu^{ \pm} \nu\right)$ & 0.1086 & $1.5 \sigma$ & $1.4 \sigma$ \\
\hline 55 & $\left\langle A_{9}\right\rangle\left(B^{0} \rightarrow K^{* 0} \mu^{+} \mu^{-}\right)^{[15,19]}$ & $6.0621 \times 10^{-5}$ & $1.4 \sigma$ & $1.4 \sigma$ \\
\hline 56 & $R_{e}^{0}$ & 20.727 & $1.5 \sigma$ & $1.4 \sigma$ \\
\hline 57 & $R_{e \mu}\left(K^{+} \rightarrow \ell^{+} \nu\right)$ & $2.4662 \times 10^{-5}$ & $2.4 \sigma$ & $1.4 \sigma$ \\
\hline 58 & $\langle\mathrm{BR}\rangle\left(B \rightarrow X_{s} e^{+} e^{-}\right)^{[14.2,25.0]}$ & $3.3821 \times 10^{-7}$ & $1.2 \sigma$ & $1.4 \sigma$ \\
\hline 59 & $R_{\tau \ell}\left(B \rightarrow D \ell^{+} \nu\right)$ & 0.31432 & $1 \sigma$ & $1.4 \sigma$ \\
\hline 60 & $S_{\phi \gamma}$ & -0.00023899 & $1.3 \sigma$ & $1.3 \sigma$ \\
\hline 61 & $\left\langle D_{P_{5}^{\prime}}^{\mu e}\right\rangle\left(B^{0} \rightarrow K^{* 0} \ell^{+} \ell^{-}\right)^{[1.0,6.0]}$ & 0.084096 & $1.2 \sigma$ & $1.3 \sigma$ \\
\hline 62 & $\mathrm{BR}\left(K_{L} \rightarrow \pi^{0} \nu \bar{\nu}\right)$ & $3.3218 \times 10^{-11}$ & $1.3 \sigma$ & $1.3 \sigma$ \\
\hline 63 & $\mathrm{BR}\left(B^{+} \rightarrow e^{+} \nu\right)$ & $9.5326 \times 10^{-12}$ & $1.3 \sigma$ & $1.3 \sigma$ \\
\hline 64 & $\frac{\langle\mathrm{BR}\rangle}{\mathrm{BR}}\left(B \rightarrow D^{*} \tau^{+} \nu\right)^{[6.0,6.5]}$ & 0.078123 & $1.3 \sigma$ & $1.3 \sigma$ \\
\hline 65 & $\left\langle\frac{d \mathrm{BR}}{d q^{2}}\right\rangle\left(B^{ \pm} \rightarrow K^{ \pm} \mu^{+} \mu^{-}\right)^{[0,2]}$ & $3.1751 \times 10^{-8}$ & $0.83 \sigma$ & $1.3 \sigma$ \\
\hline 66 & $\operatorname{BR}\left(B^{0} \rightarrow \rho^{0} \nu \bar{\nu}\right)$ & $2.0283 \times 10^{-7}$ & $1.3 \sigma$ & $1.3 \sigma$ \\
\hline 67 & $\operatorname{BR}\left(K_{S} \rightarrow \mu^{+} \mu^{-}\right)$ & $5.1934 \times 10^{-12}$ & $1.3 \sigma$ & $1.3 \sigma$ \\
\hline 68 & $\operatorname{BR}\left(K_{S} \rightarrow e^{+} e^{-}\right)$ & $1.6247 \times 10^{-16}$ & $1.3 \sigma$ & $1.3 \sigma$ \\
\hline 69 & $\mathrm{BR}\left(B^{-} \rightarrow \pi^{-} \tau^{+} e^{-}\right)$ & 0 & $1.3 \sigma$ & $1.3 \sigma$ \\
\hline 70 & $\left\langle P_{4}^{\prime}\right\rangle\left(B^{0} \rightarrow K^{* 0} \mu^{+} \mu^{-}\right)^{[2,4]}$ & -0.32702 & $1.4 \sigma$ & $1.3 \sigma$ \\
\hline 71 & $\mathrm{BR}\left(B^{0} \rightarrow K^{* 0} \nu \bar{\nu}\right)$ & $9.5415 \times 10^{-6}$ & $1.3 \sigma$ & $1.3 \sigma$ \\
\hline 72 & $\frac{\langle\mathrm{BR}\rangle}{\mathrm{BR}}\left(B \rightarrow D^{*} \tau^{+} \nu\right)^{[8.27,8.8]}$ & 0.10572 & $1.3 \sigma$ & $1.3 \sigma$ \\
\hline 73 & $\Delta M_{d}$ & $3.9784 \times 10^{-13}$ & $1.3 \sigma$ & $1.3 \sigma$ \\
\hline 74 & $\mathrm{BR}\left(\tau^{+} \rightarrow K^{+} \bar{\nu}\right)$ & 0.0071011 & $1.1 \sigma$ & $1.2 \sigma$ \\
\hline 75 & $\left\langle F_{L}\right\rangle\left(B^{0} \rightarrow K^{* 0} \mu^{+} \mu^{-}\right)^{[2,4]}$ & 0.7937 & $1.2 \sigma$ & $1.3 \sigma$ \\
\hline 76 & $\left\langle P_{5}^{\prime}\right\rangle\left(B^{0} \rightarrow K^{* 0} \mu^{+} \mu^{-}\right)^{[2.5,4]}$ & -0.45216 & $1 \sigma$ & $1.2 \sigma$ \\
\hline 77 & $\left\langle P_{4}^{\prime}\right\rangle\left(B^{0} \rightarrow K^{* 0} \mu^{+} \mu^{-}\right)^{[4,6]}$ & -0.49808 & $1.2 \sigma$ & $1.3 \sigma$ \\
\hline 78 & $\frac{\langle\mathrm{BR}\rangle}{\mathrm{BR}}\left(B \rightarrow D \tau^{+} \nu\right)^{[9.86,10.4]}$ & 0.054658 & $1.2 \sigma$ & $1.2 \sigma$ \\
\hline 79 & $a_{\tau}$ & 0.0011772 & $1.1 \sigma$ & $1.1 \sigma$ \\
\hline 80 & $\left\langle\frac{d \mathrm{BR}}{d q^{2}}\right\rangle\left(B^{0} \rightarrow K^{* 0} \mu^{+} \mu^{-}\right)^{[2,4.3]}$ & $4.0581 \times 10^{-8}$ & $0.61 \sigma$ & $1.1 \sigma$ \\
\hline 81 & $\mathrm{BR}\left(B^{+} \rightarrow K^{*+} \nu \bar{\nu}\right)$ & $1.0272 \times 10^{-5}$ & $1.1 \sigma$ & $1.1 \sigma$ \\
\hline 82 & $\left\langle P_{1}\right\rangle\left(B^{0} \rightarrow K^{* 0} e^{+} e^{-}\right)^{[0.002,1.12]}$ & 0.035971 & $1.1 \sigma$ & $1.1 \sigma$ \\
\hline 83 & $\left\langle P_{1}\right\rangle\left(B^{0} \rightarrow K^{* 0} \mu^{+} \mu^{-}\right)^{[15,19]}$ & -0.62376 & $1.1 \sigma$ & $1.1 \sigma$ \\
\hline 84 & $\frac{\langle\mathrm{BR}\rangle}{\mathrm{BR}}\left(B \rightarrow D^{*} \tau^{+} \nu\right)^{[4.0,4.5]}$ & 0.024346 & $1.1 \sigma$ & $1.1 \sigma$ \\
\hline 85 & $\left\langle F_{L}\right\rangle\left(B^{0} \rightarrow K^{* 0} \mu^{+} \mu^{-}\right)^{[1.1,2.5]}$ & 0.74205 & $0.87 \sigma$ & $1.1 \sigma$ \\
\hline 86 & $\Delta M_{s}$ & $1.2498 \times 10^{-11}$ & $1.1 \sigma$ & $1.1 \sigma$ \\
\hline 87 & $\left\langle\frac{d \mathrm{BR}}{d q^{2}}\right\rangle\left(B^{ \pm} \rightarrow K^{ \pm} \mu^{+} \mu^{-}\right)^{[2.0,3.0]}$ & $3.1564 \times 10^{-8}$ & $0.52 \sigma$ & $1.1 \sigma$ \\
\hline
\end{tabular}




\begin{tabular}{|c|c|c|c|c|}
\hline & Observable & NP prediction & NP pull & SM pull \\
\hline 88 & $\langle\mathrm{BR}\rangle\left(B \rightarrow X_{s} \mu^{+} \mu^{-}\right)^{[1.0,6.0]}$ & $1.5116 \times 10^{-6}$ & $0.88 \sigma$ & $1.1 \sigma$ \\
\hline 89 & $\left\langle\overline{S_{3}}\right\rangle\left(B_{s} \rightarrow \phi \mu^{+} \mu^{-}\right)^{[15.0,19.0]}$ & -0.20989 & $1 \sigma$ & $1 \sigma$ \\
\hline 90 & $\left\langle P_{1}\right\rangle\left(B^{0} \rightarrow K^{* 0} \mu^{+} \mu^{-}\right)^{[2,4]}$ & -0.084115 & $1.1 \sigma$ & $1.1 \sigma$ \\
\hline 91 & $\left\langle P_{5}^{\prime}\right\rangle\left(B^{0} \rightarrow K^{* 0} \mu^{+} \mu^{-}\right)^{[15,19]}$ & -0.59215 & $1 \sigma$ & $1 \sigma$ \\
\hline 92 & $\frac{\langle\mathrm{BR}\rangle}{\mathrm{BR}}\left(B \rightarrow D^{*} \tau^{+} \nu\right)^{[10.5,11.0]}$ & 0.010417 & $0.94 \sigma$ & $0.94 \sigma$ \\
\hline 93 & $\left\langle A_{7}\right\rangle\left(B^{0} \rightarrow K^{* 0} \mu^{+} \mu^{-}\right)^{[1.1,6]}$ & 0.0024641 & $0.94 \sigma$ & $0.94 \sigma$ \\
\hline 94 & $A_{\mathrm{CP}}\left(B \rightarrow X_{s+d} \gamma\right)$ & $-3.6951 \times 10^{-18}$ & $0.94 \sigma$ & $0.94 \sigma$ \\
\hline 95 & $\left\langle P_{1}\right\rangle\left(B^{0} \rightarrow K^{* 0} \mu^{+} \mu^{-}\right)^{[4,6]}$ & -0.17624 & $0.96 \sigma$ & $0.97 \sigma$ \\
\hline 96 & $\frac{\langle\mathrm{BR}\rangle}{\mathrm{BR}}\left(B \rightarrow D^{*} \tau^{+} \nu\right)^{[7.73,8.27]}$ & 0.1077 & $0.92 \sigma$ & $0.92 \sigma$ \\
\hline 97 & $\frac{\langle\mathrm{BR}\rangle}{\mathrm{BR}}\left(B \rightarrow D \tau^{+} \nu\right)^{[6.67,7.2]}$ & 0.094855 & $0.91 \sigma$ & $0.91 \sigma$ \\
\hline 98 & $\left\langle D_{P_{4}^{\prime}}^{\mu e}\right\rangle\left(B^{0} \rightarrow K^{* 0} \ell^{+} \ell^{-}\right)^{[1.0,6.0]}$ & 0.035438 & $0.84 \sigma$ & $0.91 \sigma$ \\
\hline 99 & $\langle\mathrm{BR}\rangle\left(B \rightarrow X_{s} \mu^{+} \mu^{-}\right)^{[14.2,25.0]}$ & $3.095 \times 10^{-7}$ & $0.99 \sigma$ & $0.88 \sigma$ \\
\hline 100 & $A_{\tau}$ & 0.14798 & $1.1 \sigma$ & $0.9 \sigma$ \\
\hline 101 & $\frac{\langle\mathrm{BR}\rangle}{\mathrm{BR}}\left(B \rightarrow D \tau^{+} \nu\right)^{[5.5,6.0]}$ & 0.079431 & $0.9 \sigma$ & $0.9 \sigma$ \\
\hline 102 & $\left\langle A_{7}\right\rangle\left(B^{0} \rightarrow K^{* 0} \mu^{+} \mu^{-}\right)^{[15,19]}$ & 0.0001042 & $0.89 \sigma$ & $0.89 \sigma$ \\
\hline 103 & $\operatorname{BR}\left(B^{0} \rightarrow \pi^{-} \tau^{+} \nu_{\tau}\right)$ & $8.7524 \times 10^{-5}$ & $0.91 \sigma$ & $0.96 \sigma$ \\
\hline 104 & $\left\langle\overline{S_{4}}\right\rangle\left(B_{s} \rightarrow \phi \mu^{+} \mu^{-}\right)^{[2.0,5.0]}$ & -0.14259 & $0.9 \sigma$ & $0.88 \sigma$ \\
\hline 105 & $\frac{\langle\mathrm{BR}\rangle}{\mathrm{BR}}\left(B \rightarrow D \tau^{+} \nu\right)^{[10.93,11.47]}$ & 0.024307 & $0.88 \sigma$ & $0.88 \sigma$ \\
\hline 106 & $\frac{\langle\mathrm{BR}\rangle}{\mathrm{BR}}\left(B \rightarrow D \tau^{+} \nu\right)^{[9.5,10.0]}$ & 0.058778 & $0.87 \sigma$ & $0.87 \sigma$ \\
\hline 107 & $\mathrm{BR}\left(B^{-} \rightarrow K^{-} \tau^{+} e^{-}\right)$ & 0 & $0.87 \sigma$ & $0.87 \sigma$ \\
\hline 108 & $\left\langle F_{L}\right\rangle\left(B^{0} \rightarrow K^{* 0} \mu^{+} \mu^{-}\right)^{[1,2]}$ & 0.70254 & $0.66 \sigma$ & $0.87 \sigma$ \\
\hline 109 & $\frac{\langle\mathrm{BR}\rangle}{\mathrm{BR}}\left(B \rightarrow D \tau^{+} \nu\right)^{[10.4,10.93]}$ & 0.040011 & $0.86 \sigma$ & $0.86 \sigma$ \\
\hline 110 & $\operatorname{BR}\left(\tau^{-} \rightarrow e^{-} \nu \bar{\nu}\right)$ & 0.17697 & $2.4 \sigma$ & $0.82 \sigma$ \\
\hline 111 & $S_{\psi K_{S}}$ & 0.70565 & $0.83 \sigma$ & $0.83 \sigma$ \\
\hline 112 & $\frac{\langle\mathrm{BR}\rangle}{\mathrm{BR}}\left(B \rightarrow D \tau^{+} \nu\right)^{[8.8,9.33]}$ & 0.075718 & $0.84 \sigma$ & $0.84 \sigma$ \\
\hline 113 & $\frac{\langle\mathrm{BR}\rangle}{\mathrm{BR}}\left(B \rightarrow D \tau^{+} \nu\right)^{[7.2,7.73]}$ & 0.093958 & $0.84 \sigma$ & $0.84 \sigma$ \\
\hline 114 & $\frac{\langle\mathrm{BR}\rangle}{\mathrm{BR}}\left(B \rightarrow D \tau^{+} \nu\right)^{[10.0,10.5]}$ & 0.047878 & $0.84 \sigma$ & $0.84 \sigma$ \\
\hline 115 & $\left\langle\overline{F_{L}}\right\rangle\left(B_{s} \rightarrow \phi \mu^{+} \mu^{-}\right)^{[2.0,5.0]}$ & 0.80894 & $0.86 \sigma$ & $0.87 \sigma$ \\
\hline 116 & $A_{\mathrm{FB}}^{0, c}$ & 0.074039 & $0.95 \sigma$ & $0.83 \sigma$ \\
\hline 117 & $\left\langle A_{8}\right\rangle\left(B^{0} \rightarrow K^{* 0} \mu^{+} \mu^{-}\right)^{[1.1,6]}$ & 0.0011623 & $0.83 \sigma$ & $0.83 \sigma$ \\
\hline 118 & $\mathrm{BR}\left(W^{ \pm} \rightarrow e^{ \pm} \nu\right)$ & 0.10827 & $0.73 \sigma$ & $0.82 \sigma$ \\
\hline 119 & $\frac{\langle\mathrm{BR}\rangle}{\mathrm{BR}}\left(B \rightarrow D^{*} \tau^{+} \nu\right)^{[6.67,7.2]}$ & 0.095374 & $0.82 \sigma$ & $0.82 \sigma$ \\
\hline 120 & $\frac{\langle\mathrm{BR}\rangle}{\mathrm{BR}}\left(B \rightarrow D \tau^{+} \nu\right)^{[6.0,6.5]}$ & 0.085948 & $0.82 \sigma$ & $0.82 \sigma$ \\
\hline 121 & $\left\langle P_{1}\right\rangle\left(B^{0} \rightarrow K^{* 0} \mu^{+} \mu^{-}\right)^{[1.1,2.5]}$ & 0.02995 & $0.84 \sigma$ & $0.83 \sigma$ \\
\hline 122 & $\mathrm{BR}\left(B^{+} \rightarrow \tau^{+} \nu\right)$ & $9.1574 \times 10^{-5}$ & $0.69 \sigma$ & $0.83 \sigma$ \\
\hline 123 & $\left\langle A_{9}\right\rangle\left(B^{0} \rightarrow K^{* 0} \mu^{+} \mu^{-}\right)^{[1.1,6]}$ & 0.00013215 & $0.8 \sigma$ & $0.8 \sigma$ \\
\hline 124 & $\mathrm{BR}\left(K^{+} \rightarrow \pi^{+} \nu \bar{\nu}\right)$ & $9.2404 \times 10^{-11}$ & $0.83 \sigma$ & $0.83 \sigma$ \\
\hline 125 & $\frac{\langle\mathrm{BR}\rangle}{\mathrm{BR}}\left(B \rightarrow D^{*} \tau^{+} \nu\right)^{[6.13,6.67]}$ & 0.087527 & $0.79 \sigma$ & $0.79 \sigma$ \\
\hline 126 & $\frac{\langle\mathrm{BR}\rangle}{\mathrm{BR}}\left(B \rightarrow D \tau^{+} \nu\right)^{[6.13,6.67]}$ & 0.094177 & $0.79 \sigma$ & $0.79 \sigma$ \\
\hline 127 & $\left\langle F_{L}\right\rangle\left(B^{0} \rightarrow K^{* 0} \mu^{+} \mu^{-}\right)^{[0,2]}$ & 0.36291 & $0.65 \sigma$ & $0.83 \sigma$ \\
\hline 128 & $\overline{\mathrm{BR}}\left(B_{s} \rightarrow \phi \gamma\right)$ & $4.0151 \times 10^{-5}$ & $0.8 \sigma$ & $0.8 \sigma$ \\
\hline 129 & $\left\langle A_{\mathrm{FB}}^{\ell}\right\rangle\left(\Lambda_{b} \rightarrow \Lambda \mu^{+} \mu^{-}\right)^{[15,20]}$ & -0.35201 & $0.8 \sigma$ & $0.77 \sigma$ \\
\hline 130 & $\frac{\langle\mathrm{BR}\rangle}{\mathrm{BR}}\left(B \rightarrow D^{*} \tau^{+} \nu\right)^{[8.8,9.33]}$ & 0.10126 & $0.77 \sigma$ & $0.77 \sigma$ \\
\hline 131 & $\left\langle A_{\mathrm{FB}}\right\rangle\left(B^{0} \rightarrow K^{* 0} \mu^{+} \mu^{-}\right)^{[4.3,6]}$ & 0.12076 & $0.69 \sigma$ & $0.76 \sigma$ \\
\hline 132 & $\frac{\langle\mathrm{BR}\rangle}{\mathrm{BR}}\left(B \rightarrow D \tau^{+} \nu\right)^{[7.5,8.0]}$ & 0.087076 & $0.75 \sigma$ & $0.75 \sigma$ \\
\hline 133 & $\left\langle\overline{F_{L}}\right\rangle\left(B_{s} \rightarrow \phi \mu^{+} \mu^{-}\right)^{[15.0,19.0]}$ & 0.34168 & $0.74 \sigma$ & $0.73 \sigma$ \\
\hline 134 & $\left\langle P_{1}\right\rangle\left(B^{0} \rightarrow K^{* 0} \mu^{+} \mu^{-}\right)^{[2,4.3]}$ & -0.095443 & $0.76 \sigma$ & $0.74 \sigma$ \\
\hline 135 & $\left\langle F_{L}\right\rangle\left(B^{0} \rightarrow K^{* 0} \mu^{+} \mu^{-}\right)^{[2.5,4]}$ & 0.79337 & $0.78 \sigma$ & $0.75 \sigma$ \\
\hline
\end{tabular}




\begin{tabular}{|c|c|c|c|c|}
\hline & Observable & NP prediction & NP pull & SM pull \\
\hline 136 & $\left\langle A_{\mathrm{FB}}\right\rangle\left(B^{0} \rightarrow K^{* 0} \mu^{+} \mu^{-}\right)^{[1,2]}$ & -0.16588 & $0.66 \sigma$ & $0.72 \sigma$ \\
\hline 137 & $R_{b}^{0}$ & 0.21583 & $0.7 \sigma$ & $0.73 \sigma$ \\
\hline 138 & $\frac{\langle\mathrm{BR}\rangle}{\mathrm{BR}}\left(B \rightarrow D^{*} \tau^{+} \nu\right)^{[5.5,6.0]}$ & 0.067058 & $0.72 \sigma$ & $0.72 \sigma$ \\
\hline 139 & $\frac{\langle\mathrm{BR}\rangle}{\mathrm{BR}}\left(B \rightarrow D \tau^{+} \nu\right)^{[10.5,11.0]}$ & 0.035542 & $0.71 \sigma$ & $0.71 \sigma$ \\
\hline 140 & $\frac{\langle\mathrm{BR}\rangle}{\mathrm{BR}}\left(B \rightarrow D \tau^{+} \nu\right)^{[8.5,9.0]}$ & 0.076305 & $0.71 \sigma$ & $0.71 \sigma$ \\
\hline 141 & $\frac{\langle\mathrm{BR}\rangle}{\mathrm{BR}}\left(B \rightarrow D \tau^{+} \nu\right)^{[4.0,4.53]}$ & 0.038684 & $0.69 \sigma$ & $0.69 \sigma$ \\
\hline 142 & $A_{\mathrm{FB}}^{0, e}$ & 0.016394 & $0.76 \sigma$ & $0.69 \sigma$ \\
\hline 143 & $\mathrm{BR}\left(B^{+} \rightarrow \pi^{+} \nu \bar{\nu}\right)$ & $1.2672 \times 10^{-7}$ & $0.68 \sigma$ & $0.68 \sigma$ \\
\hline 144 & $\left\langle P_{5}^{\prime}\right\rangle\left(B^{0} \rightarrow K^{* 0} \mu^{+} \mu^{-}\right)^{[4.3,6]}$ & -0.75087 & $0.71 \sigma$ & $0.65 \sigma$ \\
\hline 145 & $\operatorname{BR}\left(B^{+} \rightarrow \rho^{+} \nu \bar{\nu}\right)$ & $4.3699 \times 10^{-7}$ & $0.67 \sigma$ & $0.67 \sigma$ \\
\hline 146 & $\left\langle P_{5}^{\prime}\right\rangle\left(B^{0} \rightarrow K^{* 0} \mu^{+} \mu^{-}\right)^{[1.1,2.5]}$ & 0.1884 & $0.44 \sigma$ & $0.66 \sigma$ \\
\hline 147 & $\left\langle A_{\mathrm{FB}}\right\rangle\left(B^{0} \rightarrow K^{* 0} \mu^{+} \mu^{-}\right)^{[2,4.3]}$ & -0.041339 & $0.53 \sigma$ & $0.65 \sigma$ \\
\hline 148 & $\frac{\langle\mathrm{BR}\rangle}{\mathrm{BR}}\left(B \rightarrow D \tau^{+} \nu\right)^{[4.0,4.5]}$ & 0.035905 & $0.65 \sigma$ & $0.65 \sigma$ \\
\hline 149 & $\frac{\langle\mathrm{BR}\rangle}{\mathrm{BR}}\left(B \rightarrow D^{*} \tau^{+} \nu\right)^{[7.5,8.0]}$ & 0.098515 & $0.65 \sigma$ & $0.65 \sigma$ \\
\hline 150 & $\left\langle P_{2}\right\rangle\left(B^{0} \rightarrow K^{* 0} \mu^{+} \mu^{-}\right)^{[2.5,4]}$ & -0.11542 & $0.6 \sigma$ & $0.66 \sigma$ \\
\hline 151 & $S_{K^{*} \gamma}$ & -0.022785 & $0.63 \sigma$ & $0.63 \sigma$ \\
\hline 152 & $\mathrm{BR}\left(B^{0} \rightarrow \pi^{0} \nu \bar{\nu}\right)$ & $5.898 \times 10^{-8}$ & $0.63 \sigma$ & $0.63 \sigma$ \\
\hline 153 & $\left\langle A_{T}^{\operatorname{Im}}\right\rangle\left(B^{0} \rightarrow K^{* 0} e^{+} e^{-}\right)^{[0.002,1.12]}$ & 0.00032829 & $0.64 \sigma$ & $0.64 \sigma$ \\
\hline 154 & $\left\langle R_{\mu e}\right\rangle\left(B^{+} \rightarrow K^{*+} \ell^{+} \ell^{-}\right)^{[15.0,19.0]}$ & 0.79446 & $0.89 \sigma$ & $0.59 \sigma$ \\
\hline 155 & $\left\langle F_{L}\right\rangle\left(B^{0} \rightarrow K^{* 0} \mu^{+} \mu^{-}\right)^{[4.3,6]}$ & 0.70627 & $0.6 \sigma$ & $0.58 \sigma$ \\
\hline 156 & $\frac{\langle\mathrm{BR}\rangle}{\mathrm{BR}}\left(B \rightarrow D^{*} \tau^{+} \nu\right)^{[4.5,5.0]}$ & 0.039696 & $0.59 \sigma$ & $0.59 \sigma$ \\
\hline 157 & $A_{b}$ & 0.93475 & $0.59 \sigma$ & $0.59 \sigma$ \\
\hline 158 & $\left\langle A_{\mathrm{FB}}\right\rangle\left(B^{0} \rightarrow K^{* 0} \mu^{+} \mu^{-}\right)^{[0,2]}$ & -0.10469 & $0.62 \sigma$ & $0.62 \sigma$ \\
\hline 159 & $\operatorname{BR}\left(\tau^{-} \rightarrow e^{-} \mu^{+} e^{-}\right)$ & 0 & $0.58 \sigma$ & $0.58 \sigma$ \\
\hline 160 & $\frac{\langle\mathrm{BR}\rangle}{\mathrm{BR}}\left(B \rightarrow D \tau^{+} \nu\right)^{[8.27,8.8]}$ & 0.083993 & $0.58 \sigma$ & $0.58 \sigma$ \\
\hline 161 & $\frac{\langle\mathrm{BR}\rangle}{\mathrm{BR}}\left(B \rightarrow D^{*} \tau^{+} \nu\right)^{[10.0,10.5]}$ & 0.059012 & $0.57 \sigma$ & $0.57 \sigma$ \\
\hline 162 & $\operatorname{BR}\left(B^{-} \rightarrow K^{-} \mu^{+} \tau^{-}\right)$ & 0 & $0.57 \sigma$ & $0.57 \sigma$ \\
\hline 163 & $\frac{\langle\mathrm{BR}\rangle}{\mathrm{BR}}\left(B \rightarrow D \tau^{+} \nu\right)^{[4.53,5.07]}$ & 0.060577 & $0.56 \sigma$ & $0.56 \sigma$ \\
\hline 164 & $R_{\mu e}\left(B \rightarrow D^{*} \ell^{+} \nu\right)$ & 0.94513 & $1.4 \sigma$ & $0.56 \sigma$ \\
\hline 165 & $\left\langle P_{5}^{\prime}\right\rangle\left(B^{0} \rightarrow K^{* 0} \mu^{+} \mu^{-}\right)^{[0.04,2]}$ & 0.53161 & $0.49 \sigma$ & $0.55 \sigma$ \\
\hline 166 & $\frac{\langle\mathrm{BR}\rangle}{\mathrm{BR}}\left(B \rightarrow D^{*} \tau^{+} \nu\right)^{[4.53,5.07]}$ & 0.044486 & $0.54 \sigma$ & $0.54 \sigma$ \\
\hline 167 & $\operatorname{BR}\left(\tau^{+} \rightarrow \pi^{+} \bar{\nu}\right)$ & 0.10871 & $0.35 \sigma$ & $0.54 \sigma$ \\
\hline 168 & $A_{\mathrm{FB}}^{0, \mu}$ & 0.016318 & $0.45 \sigma$ & $0.53 \sigma$ \\
\hline 169 & $\left\langle A_{8}\right\rangle\left(B^{0} \rightarrow K^{* 0} \mu^{+} \mu^{-}\right)^{[15,19]}$ & $7.7484 \times 10^{-5}$ & $0.52 \sigma$ & $0.52 \sigma$ \\
\hline 170 & $\left\langle P_{5}^{\prime}\right\rangle\left(B^{0} \rightarrow K^{* 0} \mu^{+} \mu^{-}\right)^{[1,2]}$ & 0.32831 & $0.66 \sigma$ & $0.54 \sigma$ \\
\hline 171 & $\frac{\langle\mathrm{BR}\rangle}{\mathrm{BR}}\left(B \rightarrow D \tau^{+} \nu\right)^{[11.5,12.0]}$ & 0.0020025 & $0.51 \sigma$ & $0.51 \sigma$ \\
\hline 172 & $\operatorname{BR}\left(\tau^{-} \rightarrow \mu^{-} e^{+} \mu^{-}\right)$ & 0 & $0.51 \sigma$ & $0.51 \sigma$ \\
\hline 173 & $\mathrm{BR}\left(\pi^{+} \rightarrow e^{+} \nu\right)$ & 0.00012294 & $1.4 \sigma$ & $0.51 \sigma$ \\
\hline 174 & $\left\langle\frac{d \mathrm{BR}}{d q^{2}}\right\rangle\left(B^{0} \rightarrow K^{* 0} \mu^{+} \mu^{-}\right)^{[0,2]}$ & $7.9334 \times 10^{-8}$ & $0.69 \sigma$ & $0.54 \sigma$ \\
\hline 175 & $\operatorname{BR}\left(K_{L} \rightarrow \mu^{+} \mu^{-}\right)$ & $7.4841 \times 10^{-9}$ & $0.5 \sigma$ & $0.5 \sigma$ \\
\hline 176 & $\mathrm{BR}\left(\tau^{-} \rightarrow \mu^{-} e^{+} e^{-}\right)$ & 0 & $0.49 \sigma$ & $0.49 \sigma$ \\
\hline 177 & $\left\langle\frac{d \mathrm{BR}}{d q^{2}}\right\rangle\left(B^{0} \rightarrow K^{0} \mu^{+} \mu^{-}\right)^{[0,2]}$ & $2.9458 \times 10^{-8}$ & $0.3 \sigma$ & $0.49 \sigma$ \\
\hline 178 & $\left\langle\frac{d \mathrm{BR}}{d q^{2}}\right\rangle\left(B^{+} \rightarrow K^{*+} \mu^{+} \mu^{-}\right)^{[2.0,4.0]}$ & $4.3931 \times 10^{-8}$ & $0.78 \sigma$ & $0.5 \sigma$ \\
\hline 179 & $\operatorname{BR}\left(B^{0} \rightarrow K^{0} \nu \bar{\nu}\right)$ & $4.0717 \times 10^{-6}$ & $0.48 \sigma$ & $0.48 \sigma$ \\
\hline 180 & $\Gamma_{Z}$ & 2.4939 & $0.58 \sigma$ & $0.47 \sigma$ \\
\hline 181 & $\mathrm{BR}\left(B_{c} \rightarrow \tau^{+} \nu\right)$ & 0.024743 & $0.48 \sigma$ & $0.47 \sigma$ \\
\hline 182 & $\frac{\langle\mathrm{BR}\rangle}{\mathrm{BR}}\left(B \rightarrow D^{*} \tau^{+} \nu\right)^{[7.0,7.5]}$ & 0.094054 & $0.47 \sigma$ & $0.47 \sigma$ \\
\hline 183 & $\frac{\langle\mathrm{BR}\rangle}{\mathrm{BR}}\left(B \rightarrow D \tau^{+} \nu\right)^{[11.0,11.5]}$ & 0.020875 & $0.46 \sigma$ & $0.46 \sigma$ \\
\hline
\end{tabular}




\begin{tabular}{|c|c|c|c|c|}
\hline & Observable & NP prediction & NP pull & SM pull \\
\hline 184 & $\left\langle P_{2}\right\rangle\left(B^{0} \rightarrow K^{* 0} \mu^{+} \mu^{-}\right)^{[1.1,2.5]}$ & -0.45133 & $0.47 \sigma$ & $0.47 \sigma$ \\
\hline 185 & $\mathrm{BR}\left(B^{-} \rightarrow K^{*-} e^{+} \mu^{-}\right)$ & 0 & $0.45 \sigma$ & $0.45 \sigma$ \\
\hline 186 & $\langle\mathrm{BR}\rangle\left(B \rightarrow X_{s} e^{+} e^{-}\right)^{[1.0,6.0]}$ & $1.9486 \times 10^{-6}$ & $0.049 \sigma$ & $0.42 \sigma$ \\
\hline 187 & $\left\langle P_{4}^{\prime}\right\rangle\left(B^{0} \rightarrow K^{* 0} \mu^{+} \mu^{-}\right)^{[15,19]}$ & -0.63521 & $0.44 \sigma$ & $0.44 \sigma$ \\
\hline 188 & $\left\langle P_{4}^{\prime}\right\rangle\left(B^{0} \rightarrow K^{* 0} \mu^{+} \mu^{-}\right)^{[2.5,4]}$ & -0.3742 & $0.47 \sigma$ & $0.45 \sigma$ \\
\hline 189 & $\left\langle P_{4}^{\prime}\right\rangle\left(B^{0} \rightarrow K^{* 0} \mu^{+} \mu^{-}\right)^{[0.04,2]}$ & 0.15774 & $0.41 \sigma$ & $0.42 \sigma$ \\
\hline 190 & $\left\langle P_{4}^{\prime}\right\rangle\left(B^{0} \rightarrow K^{* 0} \mu^{+} \mu^{-}\right)^{[1.1,2.5]}$ & -0.040805 & $0.51 \sigma$ & $0.41 \sigma$ \\
\hline 191 & $\left\langle\frac{d \mathrm{BR}}{d q^{2}}\right\rangle\left(B^{ \pm} \rightarrow K^{ \pm} \mu^{+} \mu^{-}\right)^{[2,4.3]}$ & $3.1456 \times 10^{-8}$ & $0.0094 \sigma$ & $0.42 \sigma$ \\
\hline 192 & $\left\langle F_{L}\right\rangle\left(B^{0} \rightarrow K^{* 0} \mu^{+} \mu^{-}\right)^{[0.04,2]}$ & 0.36291 & $0.65 \sigma$ & $0.44 \sigma$ \\
\hline 193 & $\left\langle F_{L}\right\rangle\left(B^{0} \rightarrow K^{* 0} \mu^{+} \mu^{-}\right)^{[2,4.3]}$ & 0.78916 & $0.37 \sigma$ & $0.42 \sigma$ \\
\hline 194 & $R_{\tau}^{0}$ & 20.77 & $0.14 \sigma$ & $0.38 \sigma$ \\
\hline 195 & $\left\langle\frac{d \mathrm{BR}}{d q^{2}}\right\rangle\left(B^{0} \rightarrow K^{0} \mu^{+} \mu^{-}\right)^{[2,4.3]}$ & $2.9181 \times 10^{-8}$ & $0.21 \sigma$ & $0.39 \sigma$ \\
\hline 196 & $\left\langle R_{\mu e}\right\rangle\left(B^{0} \rightarrow K^{* 0} \ell^{+} \ell^{-}\right)^{[0.1,8.0]}$ & 0.82242 & $0.19 \sigma$ & $0.37 \sigma$ \\
\hline 197 & $\left\langle R_{\mu e}\right\rangle\left(B^{0} \rightarrow K^{* 0} \ell^{+} \ell^{-}\right)^{[15.0,19.0]}$ & 0.79447 & $0.89 \sigma$ & $0.36 \sigma$ \\
\hline 198 & $\left\langle P_{2}\right\rangle\left(B^{0} \rightarrow K^{* 0} \mu^{+} \mu^{-}\right)^{[15,19]}$ & 0.37143 & $0.32 \sigma$ & $0.36 \sigma$ \\
\hline 199 & $\left\langle P_{1}\right\rangle\left(B^{0} \rightarrow K^{* 0} \mu^{+} \mu^{-}\right)^{[2.5,4]}$ & -0.10663 & $0.33 \sigma$ & $0.34 \sigma$ \\
\hline 200 & $\left\langle A_{\mathrm{FB}}^{h}\right\rangle\left(\Lambda_{b} \rightarrow \Lambda \mu^{+} \mu^{-}\right)^{[15,20]}$ & -0.31822 & $0.31 \sigma$ & $0.31 \sigma$ \\
\hline 201 & $\frac{\langle\mathrm{BR}\rangle}{\mathrm{BR}}\left(B \rightarrow D \tau^{+} \nu\right)^{[6.5,7.0]}$ & 0.089095 & $0.34 \sigma$ & $0.34 \sigma$ \\
\hline 202 & $A_{\mu}$ & 0.14716 & $0.34 \sigma$ & $0.34 \sigma$ \\
\hline 203 & $\frac{\langle\mathrm{BR}\rangle}{\mathrm{BR}}\left(B \rightarrow D^{*} \tau^{+} \nu\right)^{[9.86,10.4]}$ & 0.071012 & $0.33 \sigma$ & $0.33 \sigma$ \\
\hline 204 & $\overline{\mathrm{BR}}\left(B_{s} \rightarrow \tau^{+} \tau^{-}\right)$ & $9.0721 \times 10^{-7}$ & $0.33 \sigma$ & $0.33 \sigma$ \\
\hline 205 & $\left\langle P_{1}\right\rangle\left(B^{0} \rightarrow K^{* 0} \mu^{+} \mu^{-}\right)^{[0.04,2]}$ & 0.043716 & $0.32 \sigma$ & $0.32 \sigma$ \\
\hline 206 & $\operatorname{BR}\left(\bar{B}^{0} \rightarrow \bar{K}^{* 0} \mu^{+} e^{-}\right)$ & 0 & $0.3 \sigma$ & $0.3 \sigma$ \\
\hline 207 & $\frac{\langle\mathrm{BR}\rangle}{\mathrm{BR}}\left(B \rightarrow D^{*} \tau^{+} \nu\right)^{[8.5,9.0]}$ & 0.098623 & $0.3 \sigma$ & $0.3 \sigma$ \\
\hline 208 & $\mathrm{BR}\left(B^{-} \rightarrow K^{-} \tau^{+} \mu^{-}\right)$ & 0 & $0.29 \sigma$ & $0.29 \sigma$ \\
\hline 209 & $\frac{\langle\mathrm{BR}\rangle}{\mathrm{BR}}\left(B \rightarrow D \tau^{+} \nu\right)^{[4.5,5.0]}$ & 0.054469 & $0.27 \sigma$ & $0.27 \sigma$ \\
\hline 210 & $\left\langle\frac{d \mathrm{BR}}{d q^{2}}\right\rangle\left(B^{+} \rightarrow K^{*+} \mu^{+} \mu^{-}\right)^{[0,2]}$ & $8.3078 \times 10^{-8}$ & $0.14 \sigma$ & $0.21 \sigma$ \\
\hline 211 & $\mathrm{BR}\left(B^{-} \rightarrow K^{*-} \mu^{+} e^{-}\right)$ & 0 & $0.25 \sigma$ & $0.25 \sigma$ \\
\hline 212 & $\left\langle\overline{S_{3}}\right\rangle\left(B_{s} \rightarrow \phi \mu^{+} \mu^{-}\right)^{[2.0,5.0]}$ & -0.0078565 & $0.27 \sigma$ & $0.27 \sigma$ \\
\hline 213 & $\mathrm{BR}\left(B^{+} \rightarrow \mu^{+} \nu\right)$ & $3.8612 \times 10^{-7}$ & $0.26 \sigma$ & $0.24 \sigma$ \\
\hline 214 & $\left\langle P_{5}^{\prime}\right\rangle\left(B^{0} \rightarrow K^{* 0} \mu^{+} \mu^{-}\right)^{[2,4.3]}$ & -0.39945 & $0.37 \sigma$ & $0.24 \sigma$ \\
\hline 215 & $x_{12}^{\operatorname{Im}, D}$ & $-3.4139 \times 10^{-20}$ & $0.25 \sigma$ & $0.25 \sigma$ \\
\hline 216 & $\frac{\langle\mathrm{BR}\rangle}{\mathrm{BR}}\left(B \rightarrow D^{*} \tau^{+} \nu\right)^{[6.5,7.0]}$ & 0.087179 & $0.22 \sigma$ & $0.22 \sigma$ \\
\hline 217 & $\frac{\langle\mathrm{BR}\rangle}{\mathrm{BR}}\left(B \rightarrow D^{*} \tau^{+} \nu\right)^{[9.33,9.86]}$ & 0.0907 & $0.22 \sigma$ & $0.22 \sigma$ \\
\hline 218 & $\frac{\langle\mathrm{BR}\rangle}{\mathrm{BR}}\left(B \rightarrow D \tau^{+} \nu\right)^{[11.47,12.0]}$ & 0.0026758 & $0.22 \sigma$ & $0.22 \sigma$ \\
\hline 219 & $\mathrm{BR}\left(B^{-} \rightarrow K^{-} e^{+} \tau^{-}\right)$ & 0 & $0.2 \sigma$ & $0.2 \sigma$ \\
\hline 220 & $\left\langle P_{5}^{\prime}\right\rangle\left(B^{0} \rightarrow K^{* 0} \mu^{+} \mu^{-}\right)^{[2,4]}$ & -0.35661 & $0.11 \sigma$ & $0.26 \sigma$ \\
\hline 221 & $\mathrm{BR}\left(B^{-} \rightarrow \pi^{-} \mu^{+} \tau^{-}\right)$ & 0 & $0.18 \sigma$ & $0.18 \sigma$ \\
\hline 222 & $\mathrm{BR}\left(B^{0} \rightarrow K^{* 0} \gamma\right)$ & $4.1772 \times 10^{-5}$ & $0.18 \sigma$ & $0.18 \sigma$ \\
\hline 223 & $S_{\psi \phi}$ & 0.03873 & $0.18 \sigma$ & $0.18 \sigma$ \\
\hline 224 & $\mathrm{BR}\left(B^{0} \rightarrow \mu^{+} \mu^{-}\right)$ & $1.0315 \times 10^{-10}$ & $0.034 \sigma$ & $0.17 \sigma$ \\
\hline 225 & $\frac{\langle\mathrm{BR}\rangle}{\mathrm{BR}}\left(B \rightarrow D^{*} \tau^{+} \nu\right)^{[5.6,6.13]}$ & 0.073949 & $0.16 \sigma$ & $0.16 \sigma$ \\
\hline 226 & $\Gamma_{W}$ & 2.0917 & $0.16 \sigma$ & $0.16 \sigma$ \\
\hline 227 & $\left\langle\frac{d \mathrm{BR}}{d q^{2}}\right\rangle\left(B^{0} \rightarrow K^{* 0} \mu^{+} \mu^{-}\right)^{[1,2]}$ & $4.4936 \times 10^{-8}$ & $0.2 \sigma$ & $0.17 \sigma$ \\
\hline 228 & $\frac{\langle\mathrm{BR}\rangle}{\mathrm{BR}}\left(B \rightarrow D^{*} \tau^{+} \nu\right)^{[9.5,10.0]}$ & 0.081195 & $0.16 \sigma$ & $0.16 \sigma$ \\
\hline 229 & $\frac{\langle\mathrm{BR}\rangle}{\mathrm{BR}}\left(B \rightarrow D \tau^{+} \nu\right)^{[7.0,7.5]}$ & 0.089338 & $0.16 \sigma$ & $0.16 \sigma$ \\
\hline 230 & $\left\langle P_{1}\right\rangle\left(B^{0} \rightarrow K^{* 0} \mu^{+} \mu^{-}\right)^{[1,2]}$ & 0.047436 & $0.13 \sigma$ & $0.14 \sigma$ \\
\hline 231 & $\frac{\langle\mathrm{BR}\rangle}{\mathrm{BR}}\left(B \rightarrow D^{*} \tau^{+} \nu\right)^{[8.0,8.5]}$ & 0.10022 & $0.15 \sigma$ & $0.15 \sigma$ \\
\hline
\end{tabular}




\begin{tabular}{|c|c|c|c|c|}
\hline & Observable & NP prediction & NP pull & SM pull \\
\hline 232 & $\frac{\langle\mathrm{BR}\rangle}{\mathrm{BR}}\left(B \rightarrow D \tau^{+} \nu\right)^{[5.6,6.13]}$ & 0.086109 & $0.14 \sigma$ & $0.14 \sigma$ \\
\hline 233 & $\sigma_{\text {trident }} / \sigma_{\text {trident }}^{\mathrm{SM}}$ & 1.0035 & $0.14 \sigma$ & $0.13 \sigma$ \\
\hline 234 & $\frac{\langle\mathrm{BR}\rangle}{\mathrm{BR}}\left(B \rightarrow D \tau^{+} \nu\right)^{[8.0,8.5]}$ & 0.082641 & $0.12 \sigma$ & $0.12 \sigma$ \\
\hline 235 & $\frac{\langle\mathrm{BR}\rangle}{\mathrm{BR}}\left(B \rightarrow D \tau^{+} \nu\right)^{[5.0,5.5]}$ & 0.069057 & $0.12 \sigma$ & $0.12 \sigma$ \\
\hline 236 & $\left\langle F_{L}\right\rangle\left(B^{0} \rightarrow K^{* 0} \mu^{+} \mu^{-}\right)^{[15,19]}$ & 0.3406 & $0.098 \sigma$ & $0.11 \sigma$ \\
\hline 237 & $\left\langle R_{\mu e}\right\rangle\left(B^{+} \rightarrow K^{*+} \ell^{+} \ell^{-}\right)^{[0.1,8.0]}$ & 0.82185 & $0.41 \sigma$ & $0.1 \sigma$ \\
\hline 238 & $\mathrm{BR}\left(\tau^{-} \rightarrow e^{-} e^{+} e^{-}\right)$ & 0 & $0.1 \sigma$ & $0.1 \sigma$ \\
\hline 239 & $A_{c}$ & 0.6677 & $0.085 \sigma$ & $0.092 \sigma$ \\
\hline 240 & $\mathrm{BR}\left(B \rightarrow X_{s} \gamma\right)$ & 0.0003291 & $0.086 \sigma$ & $0.086 \sigma$ \\
\hline 241 & $\left\langle\frac{d \mathrm{BR}}{d q^{2}}\right\rangle\left(\Lambda_{b} \rightarrow \Lambda \mu^{+} \mu^{-}\right)^{[1.1,6]}$ & $9.362 \times 10^{-9}$ & $0.056 \sigma$ & $0.065 \sigma$ \\
\hline 242 & $\left\langle D_{P_{4}^{\prime}}^{\mu e}\right\rangle\left(B^{0} \rightarrow K^{* 0} \ell^{+} \ell^{-}\right)^{[14.18,19.0]}$ & -0.00016124 & $0.072 \sigma$ & $0.072 \sigma$ \\
\hline 243 & $\frac{\langle\mathrm{BR}\rangle}{\mathrm{BR}}\left(B \rightarrow D^{*} \tau^{+} \nu\right)^{[4.0,4.53]}$ & 0.026298 & $0.068 \sigma$ & $0.068 \sigma$ \\
\hline 244 & $\frac{\langle\mathrm{BR}\rangle}{\mathrm{BR}}\left(B \rightarrow D \tau^{+} \nu\right)^{[5.07,5.6]}$ & 0.075356 & $0.067 \sigma$ & $0.067 \sigma$ \\
\hline 245 & $\frac{\langle\mathrm{BR}\rangle}{\mathrm{BR}}\left(B \rightarrow D \tau^{+} \nu\right)^{[9.33,9.86]}$ & 0.065584 & $0.048 \sigma$ & $0.048 \sigma$ \\
\hline 246 & & 0.17222 & $0.04 \sigma$ & $0.041 \sigma$ \\
\hline 247 & $R_{c}^{0}$ & $4.245 \times 10^{-5}$ & $0.04 \sigma$ & $0.04 \sigma$ \\
\hline 248 & $\frac{\langle\mathrm{BR}\rangle}{\mathrm{BR}}\left(B \rightarrow D^{*} \tau^{+} \nu\right)^{[9.0,9.5]}$ & 0.09285 & $0.028 \sigma$ & $0.028 \sigma$ \\
\hline 249 & $\left\langle\frac{d \mathrm{BR}}{d q^{2}}\right\rangle\left(B^{+} \rightarrow K^{*+} \mu^{+} \mu^{-}\right)^{[2,4.3]}$ & $4.4175 \times 10^{-8}$ & $0.13 \sigma$ & $0.0019 \sigma$ \\
\hline 250 & $\frac{\langle\mathrm{BRR}\rangle}{\mathrm{BR}}\left(B \rightarrow D^{*} \tau^{+} \nu\right)^{[5.0,5.5]}$ & 0.054155 & $0.0066 \sigma$ & $0.0066 \sigma$ \\
\hline 251 & $\mathrm{BR}\left(B^{0} \rightarrow \tau^{+} \tau^{-}\right)$ & $2.7877 \times 10^{-8}$ & $0.0051 \sigma$ & $0.0047 \sigma$ \\
\hline & & & & \\
\hline & & & \\
\hline
\end{tabular}

\section{References}

1. R. Aaij et al. [LHCb Collaboration], JHEP 1406, 133 (2014). https://doi.org/10.1007/JHEP06(2014)133 [arXiv:1403.8044 [hep-ex]]

2. R. Aaij et al. [LHCb Collaboration], Phys. Rev. Lett. 113, 151601 (2014). https://doi.org/10.1103/PhysRevLett.113.151601 [arXiv:1406.6482 [hep-ex]]

3. R. Aaij et al. [LHCb Collaboration], JHEP 1509, 179 (2015). https://doi.org/10.1007/JHEP09(2015)179 [arXiv:1506.08777 [hep-ex]]

4. R. Aaij et al. [LHCb Collaboration], JHEP 1602, 104 (2016). https://doi.org/10.1007/JHEP02(2016)104 [arXiv:1512.04442 [hep-ex]]

5. R. Aaij et al. [LHCb Collaboration], JHEP 1611, 047 (2016). Erratum: [JHEP 1704, 142 (2017)] https://doi.org/10.1007/JHEP11(2016)047, https:// doi.org/10.1007/JHEP04(2017)142 [arXiv:1606.04731 [hep-ex]]

6. V. Khachatryan et al. [CMS and LHCb Collaborations], Nature 522, 68 (2015). https://doi.org/10.1038/nature14474 [arXiv:1411.4413 [hep-ex]]

7. R. Aaij et al. [LHCb Collaboration], Phys. Rev. Lett. 115(11), 111803 (2015). Erratum: [Phys. Rev. Lett. 115(15), 159901 (2015)] https://doi.org/10. 1103/PhysRevLett.115.159901, https://doi.org/10.1103/PhysRevLett.115.111803 [arXiv:1506.08614 [hep-ex]]

8. R. Aaij et al. [LHCb Collaboration], Eur. Phys. J. C 77(3), 161 (2017). https://doi.org/10.1140/epjc/s10052-017-4703-2 [arXiv:1612.06764 [hep-ex]]

9. R. Aaij et al. [LHCb Collaboration], Phys. Rev. Lett. 118(19), 191801 (2017). https://doi.org/10.1103/PhysRevLett.118.191801 [arXiv:1703.05747 [hep-ex]]

10. R. Aaij et al. [LHCb Collaboration], Phys. Rev. Lett. 118(25), 251802 (2017). https://doi.org/10.1103/PhysRevLett.118.251802 [arXiv:1703.02508 [hep-ex]]

11. R. Aaij et al. [LHCb Collaboration], JHEP 1708, 055 (2017). https://doi.org/10.1007/JHEP08(2017)055 [arXiv:1705.05802 [hep-ex]]

12. R. Aaij et al. [LHCb Collaboration], Phys. Rev. Lett. 120(17), 171802 (2018). https://doi.org/10.1103/PhysRevLett.120.171802 [arXiv:1708.08856 [hep-ex]]

13. R. Aaij et al. [LHCb Collaboration], JHEP 07, 020 (2018). https://doi.org/10.1007/JHEP07(2018)020 [arXiv:1804.07167 [hep-ex]]

14. R. Aaij et al. [LHCb Collaboration], Phys. Rev. Lett. 122(19), 191801 (2019). https://doi.org/10.1103/PhysRevLett.122.191801 [arXiv:1903.09252 [hep-ex]]

15. R. Aaij et al. [LHCb Collaboration]. [arXiv:2103.11769 [hep-ex]]

16. J.P. Lees et al. [BaBar], Phys. Rev. Lett. 109, 101802 (2012). https://doi.org/10.1103/PhysRevLett.109.101802 [arxiv:1205.5442 [hep-ex]]

17. A. Abdesselam et al. [Belle Collaboration], arXiv:1702.01521 [hep-ex]

18. E. Waheed et al. [Belle Collaboration], Phys. Rev. D 100(5), 052007 (2019). [erratum: Phys. Rev. D 103(7), 079901 (2021)] https://doi.org/10.1103/ PhysRevD.100.052007 [arXiv:1809.03290 [hep-ex]]

19. A. Abdesselam et al. [Belle Collaboration]. arXiv:1904.02440 [hep-ex]

20. A. Abdesselam et al. [Belle Collaboration]. [arXiv:1904.08794 [hep-ex]]

21. S. Choudhury et al. [Belle Collaboration], JHEP 2103, 105 (2021). https://doi.org/10.1007/JHEP03(2021)105 [arXiv:1908.01848 [hep-ex]]

22. M. Jung, D.M. Straub, JHEP 01, 009 (2019). https://doi.org/10.1007/JHEP01(2019)009 [arXiv:1801.01112 [hep-ph]]

23. Y.S. Amhis et al. [HFLAV], Eur. Phys. J. C 81(3), 226 (2021). https://doi.org/10.1140/epjc/s10052-020-8156-7 [arXiv:1909.12524 [hep-ex]]

24. Y. Amhis et al. [HFLAV], Eur. Phys. J. C 77 (12), 895 (2017). https://doi.org/10.1140/epjc/s10052-017-5058-4 [arXiv:1612.07233 [hep-ex]]

25. S. Descotes-Genon, J. Matias, M. Ramon, J. Virto, JHEP 01, 048 (2013). https://doi.org/10.1007/JHEP01(2013)048 [arXiv:1207.2753 [hep-ph]]

26. G. Hiller, F. Kruger, Phys. Rev. D 69, 074020 (2004). https://doi.org/10.1103/PhysRevD.69.074020 [arXiv:hep-ph/0310219 [hep-ph]]

27. M. Bordone, G. Isidori, A. Pattori, Eur. Phys. J. C 76(8), 440 (2016). https://doi.org/10.1140/epjc/s10052-016-4274-7 [arXiv:1605.07633 [hep-ph]]

28. W. Altmannshofer, C. Niehoff, P. Stangl, D.M. Straub, Eur. Phys. J. C 77(6), 377. https://doi.org/10.1140/epjc/s10052-017-4952-0 [arXiv:1703.09189 [hep-ph]]

29. G. Hiller, M. Schmaltz, Phys. Rev. D 90, 054014 (2014). https://doi.org/10.1103/PhysRevD.90.054014 [arXiv:1408.1627 [hep-ph]] 
30. G. Hiller, M. Schmaltz, JHEP 1502, 055 (2015) https://doi.org/10.1007/JHEP02(2015)055 [arXiv:1411.4773 [hep-ph]]

31. A. Crivellin, G. D’Ambrosio, J. Heeck, Phys. Rev. D 91(7), 075006 (2015). https://doi.org/10.1103/PhysRevD.91.075006 [arXiv:1503.03477 [hep-ph]]

32. A. Crivellin, L. Hofer, J. Matias, U. Nierste, S. Pokorski, J. Rosiek, Phys. Rev. D 92(5), 054013 (2015). https://doi.org/10.1103/PhysRevD.92.054013 [arXiv:1504.07928 [hep-ph]]

33. T. Hurth, F. Mahmoudi, S. Neshatpour, Nucl. Phys. B 909, 737 (2016). https://doi.org/10.1016/j.nuclphysb.2016.05.022 [arXiv:1603.00865 [hep-ph]]

34. B. Capdevila, S. Descotes-Genon, L. Hofer, J. Matias, JHEP 1704, 016 (2017). https://doi.org/10.1007/JHEP04(2017)016 [arXiv:1701.08672 [hep-ph]]

35. V.G. Chobanova, T. Hurth, F. Mahmoudi, D. Martinez Santos, S. Neshatpour, JHEP 1707, 025 (2017). https://doi.org/10.1007/JHEP07(2017)025 [arXiv:1702.02234 [hep-ph]]

36. W. Altmannshofer, C. Niehoff, D.M. Straub, JHEP 1705, 076 (2017). https://doi.org/10.1007/JHEP05(2017)076 [arXiv:1702.05498 [hep-ph]]

37. W. Altmannshofer, P. Stangl, D.M. Straub, Phys. Rev. D 96(5), 055008 (2017). https://doi.org/10.1103/PhysRevD.96.055008 [arXiv:1704.05435 [hep-ph]]

38. G. Hiller, I. Nisandzic, Phys. Rev. D 96(3), 035003 (2017). https://doi.org/10.1103/PhysRevD.96.035003 [arXiv:1704.05444 [hep-ph]]

39. L.S. Geng, B. Grinstein, S. Jäger, J. Martin Camalich, X.L. Ren, R.X. Shi, Phys. Rev. D 96(9), 093006 (2017). https://doi.org/10.1103/PhysRevD.96. 093006 [arXiv:1704.05446 [hep-ph]]

40. M. Ciuchini, A.M. Coutinho, M. Fedele, E. Franco, A. Paul, L. Silvestrini, M. Valli, Eur. Phys. J. C 77(10), 688 (2017). https://doi.org/10.1140/epjc/ s10052-017-5270-2 [arXiv:1704.05447 [hep-ph]]

41. J. Alda, J. Guasch, S. Penaranda, Eur. Phys. J. C 79(7), 588 (2019). https://doi.org/10.1140/epjc/s10052-019-7092-x [arXiv:1805.03636 [hep-ph]]

42. R. Coy, M. Frigerio, F. Mescia, O. Sumensari, Eur. Phys. J. C 80(1), 52 (2020). https://doi.org/10.1140/epjc/s10052-019-7581-y [arXiv:1909.08567 [hep-ph]]

43. A. Celis, M. Jung, X.Q. Li, A. Pich, Phys. Lett. B 771, 168-179 (2017). https://doi.org/10.1016/j.physletb.2017.05.037 [arXiv:1612.07757 [hep-ph]]

44. M.A. Ivanov, J.G. Körner, C.T. Tran, Phys. Rev. D 95(3), 036021 (2017). https://doi.org/10.1103/PhysRevD.95.036021 [arXiv:1701.02937 [hep-ph]]

45. W. Altmannshofer, P.S. Bhupal Dev, A. Soni, Phys. Rev. D 96(9), 095010 (2017). https://doi.org/10.1103/PhysRevD.96.095010 [arXiv:1704.06659 [hep-ph]]

46. D. Bigi, P. Gambino, S. Schacht, JHEP 11, 061 (2017). https://doi.org/10.1007/JHEP11(2017)061 [arXiv:1707.09509 [hep-ph]]

47. S. Iguro, K. Tobe, Nucl. Phys. B 925, 560-606 (2017). https://doi.org/10.1016/j.nuclphysb.2017.10.014 [arXiv:1708.06176 [hep-ph]]

48. A.K. Alok, D. Kumar, J. Kumar, S. Kumbhakar, S.U. Sankar, JHEP 09, 152 (2018). https://doi.org/10.1007/JHEP09(2018)152 [arXiv:1710.04127 [hep-ph]]

49. A. Azatov, D. Bardhan, D. Ghosh, F. Sgarlata, E. Venturini, JHEP 11, 187 (2018). https://doi.org/10.1007/JHEP11(2018)187 [arXiv:1805.03209 [hep-ph]]

50. S. Bhattacharya, S. Nandi, S. Kumar Patra, Eur. Phys. J. C 79(3), 268 (2019). https://doi.org/10.1140/epjc/s10052-019-6767-7 [arXiv:1805.08222 [hep-ph]]

51. Z.R. Huang, Y. Li, C.D. Lu, M.A. Paracha, C. Wang, Phys. Rev. D 98(9), 095018 (2018). https://doi.org/10.1103/PhysRevD.98.095018 [arXiv: 1808.03565 [hep-ph]]

52. M. Blanke, A. Crivellin, S. de Boer, T. Kitahara, M. Moscati, U. Nierste, I. Nišandžić, Phys. Rev. D 99(7), 075006 (2019). https://doi.org/10.1103/ PhysRevD.99.075006 [arXiv:1811.09603 [hep-ph]]

53. C. Murgui, A. Peñuelas, M. Jung, A. Pich, JHEP 09, 103 (2019). https://doi.org/10.1007/JHEP09(2019)103 [arXiv:1904.09311 [hep-ph]]

54. M. Blanke, A. Crivellin, T. Kitahara, M. Moscati, U. Nierste, I. Nišandžić, Phys. Rev. D 100(3), 035035 (2019). https://doi.org/10.1103/PhysRevD. 100.035035 [arXiv: 1905.08253 [hep-ph]]

55. X.Q. Hu, S.P. Jin, Z.J. Xiao, Chin. Phys. C 44(5), 053102 (2020). https://doi.org/10.1088/1674-1137/44/5/053102 [arXiv:1912.03981 [hep-ph]]

56. R. Mandal, C. Murgui, A. Peñuelas, A. Pich, JHEP 08(08), 022 (2020). https://doi.org/10.1007/JHEP08(2020)022 [arXiv:2004.06726 [hep-ph]]

57. S. Iguro, M. Takeuchi, R. Watanabe, Eur. Phys. J. C 81(5), 406 (2021). https://doi.org/10.1140/epjc/s10052-021-09125-5 [arXiv:2011.02486 [hep-ph]]

58. D. Bečirević, F. Jaffredo, A. Peñuelas, O. Sumensari, JHEP 05, 175 (2021). https://doi.org/10.1007/JHEP05(2021)175 [arXiv:2012.09872 [hep-ph]]

59. B. Bhattacharya, A. Datta, D. London, S. Shivashankara, Phys. Lett. B 742, 370-374 (2015). https://doi.org/10.1016/j.physletb.2015.02.011 [arXiv:1412.7164 [hep-ph]]

60. G. Hiller, D. Loose, K. Schönwald, JHEP 12, 027 (2016). https://doi.org/10.1007/JHEP12(2016)027 [arXiv:1609.08895 [hep-ph]]

61. B. Bhattacharya, A. Datta, J.P. Guévin, D. London, R. Watanabe, JHEP 01, 015 (2017). https://doi.org/10.1007/JHEP01(2017)015 [arXiv:1609.09078 [hep-ph]]

62. Y. Cai, J. Gargalionis, M.A. Schmidt, R.R. Volkas, JHEP 10, 047 (2017). https://doi.org/10.1007/JHEP10(2017)047 [arXiv:1704.05849 [hep-ph]]

63. A.K. Alok, D. Kumar, J. Kumar, R. Sharma, Eur. Phys. J. C 79(8), 707 (2019). https://doi.org/10.1140/epjc/s10052-019-7219-0 [arXiv:1704.07347 [hep-ph]]

64. F. Feruglio, P. Paradisi, A. Pattori, JHEP 1709, 061 (2017). https://doi.org/10.1007/JHEP09(2017)061 [arXiv:1705.00929 [hep-ph]]

65. D. Buttazzo, A. Greljo, G. Isidori, D. Marzocca, JHEP 11, 044 (2017). https://doi.org/10.1007/JHEP11(2017)044 [arXiv:1706.07808 [hep-ph]]

66. L. Di Luzio, A. Greljo, M. Nardecchia, Phys. Rev. D 96(11), 115011 (2017). https://doi.org/10.1103/PhysRevD.96.115011 [arXiv:1708.08450 [hep$\mathrm{ph}]]$

67. M. Bordone, C. Cornella, J. Fuentes-Martin, G. Isidori, Phys. Lett. B 779, 317-323 (2018). https://doi.org/10.1016/j.physletb.2018.02.011 [arXiv: 1712.01368 [hep-ph]]

68. M. Blanke, A. Crivellin, Phys. Rev. Lett. 121(1), 011801 (2018). https://doi.org/10.1103/PhysRevLett.121.011801 [arXiv:1801.07256 [hep-ph]]

69. D. Bečirević, I. Doršner, S. Fajfer, N. Košnik, D.A. Faroughy, O. Sumensari, Phys. Rev. D 98(5), 055003 (2018). https://doi.org/10.1103/PhysRevD. 98.055003 [arXiv:1806.05689 [hep-ph]]

70. J. Kumar, D. London, R. Watanabe, Phys. Rev. D 99(1), 015007 (2019). https://doi.org/10.1103/PhysRevD.99.015007 [arXiv:1806.07403 [hep-ph]]

71. A. Angelescu, D. Bečirević, D.A. Faroughy, O. Sumensari, JHEP 10, 183 (2018). https://doi.org/10.1007/JHEP10(2018)183 [arXiv:1808.08179 [hep-ph]]

72. S. Bifani, S. Descotes-Genon, A. Romero Vidal, M.H. Schune, J. Phys. G 46(2), 023001 (2019). https://doi.org/10.1088/1361-6471/aaf5de [arXiv: 1809.06229 [hep-ex]]

73. S. Saad, A. Thapa, Phys. Rev. D 102(1), 015014 (2020). https://doi.org/10.1103/PhysRevD.102.015014 [arXiv:2004.07880 [hep-ph]]

74. K.S. Babu, P.S.B. Dev, S. Jana, A. Thapa, JHEP 03, 179 (2021). https://doi.org/10.1007/JHEP03(2021)179 [arXiv:2009.01771 [hep-ph]]

75. B. Capdevila, A. Crivellin, S. Descotes-Genon, J. Matias, J. Virto, JHEP 1801, 093 (2018). https://doi.org/10.1007/JHEP01(2018)093 [arXiv: 1704.05340 [hep-ph]]

76. A. Celis, J. Fuentes-Martin, A. Vicente, J. Virto, Phys. Rev. D 96(3), 035026 (2017). https://doi.org/10.1103/PhysRevD.96.035026 [arXiv:1704.05672 [hep-ph]] 
77. J.E. Camargo-Molina, A. Celis, D.A. Faroughy, Phys. Lett. B 784, 284-293 (2018). https://doi.org/10.1016/j.physletb.2018.07.051 [arXiv:1805.04917 [hep-ph]]

78. A.K. Alok, B. Bhattacharya, A. Datta, D. Kumar, J. Kumar, D. London, Phys. Rev. D 96(9), 095009 (2017). https://doi.org/10.1103/PhysRevD.96. 095009 [arXiv:1704.07397 [hep-ph]]

79. A. Datta, J. Kumar, D. London, Phys. Lett. B 797, 134858 (2019). https://doi.org/10.1016/j.physletb.2019.134858 [arXiv:1903.10086 [hep-ph]]

80. J. Aebischer, W. Altmannshofer, D. Guadagnoli, M. Reboud, P. Stangl, D.M. Straub, Eur. Phys. J. C 80(3), 252 (2020). https://doi.org/10.1140/epjc/ s10052-020-7817-x [arXiv:1903.10434 [hep-ph]], and references therein

81. R. Aoude, T. Hurth, S. Renner, W. Shepherd, JHEP 12, 113 (2020). https://doi.org/10.1007/JHEP12(2020)113 [arXiv:2003.05432 [hep-ph]]

82. A.J. Buras, Contribution to: Les Houches Summer School in Theoretical Physics, Session 68: Probing the Standard Model of Particle Interactions, 281-539 [arXiv:hep-ph/9806471 [hep-ph]]

83. J. Aebischer, A. Crivellin, M. Fael, C. Greub, JHEP 05, 037 (2016). https://doi.org/10.1007/JHEP05(2016)037 [arXiv:1512.02830 [hep-ph]]

84. J. Aebischer, M. Fael, C. Greub, J. Virto, JHEP 09, 158 (2017). https://doi.org/10.1007/JHEP09(2017)158 [arXiv:1704.06639 [hep-ph]]

85. M. Tanaka, R. Watanabe, Phys. Rev. D 87(3), 034028 (2013). https://doi.org/10.1103/PhysRevD.87.034028 [arXiv:1212.1878 [hep-ph]]

86. B. Grzadkowski, M. Iskrzynski, M. Misiak, J. Rosiek, JHEP 10, 085 (2010). https://doi.org/10.1007/JHEP10(2010)085 [arXiv:1008.4884 [hep-ph]]

87. A. Dedes, W. Materkowska, M. Paraskevas, J. Rosiek, K. Suxho, JHEP 06, 143 (2017). https://doi.org/10.1007/JHEP06(2017)143 [arXiv:1704.03888 [hep-ph]]

88. R. Barbieri, G. Isidori, J. Jones-Perez, P. Lodone, D.M. Straub, Eur. Phys. J. C 71, 1725 (2011). https://doi.org/10.1140/epjc/s10052-011-1725-z. [arXiv:1105.2296 [hep-ph]]

89. R. Barbieri, D. Buttazzo, F. Sala, D.M. Straub, JHEP 07, 181 (2012). https://doi.org/10.1007/JHEP07(2012)181 [arXiv:1203.4218 [hep-ph]]

90. J.A. Aguilar-Saavedra et al., arXiv:1802.07237 [hep-ph]

91. J. Aebischer, J. Kumar, D.M. Straub, Eur. Phys. J. C 78(12), 1026 (2018). https://doi.org/10.1140/epjc/s10052-018-6492-7 [arXiv:1804.05033 [hep$\mathrm{ph}]]$

92. E.E. Jenkins, A.V. Manohar, P. Stoffer, JHEP 03, 016 (2018). https://doi.org/10.1007/JHEP03(2018)016 [arXiv:1709.04486 [hep-ph]]

93. S. Descotes-Genon, A. Falkowski, M. Fedele, M. González-Alonso, J. Virto, JHEP 05, 172 (2019). https://doi.org/10.1007/ JHEP05(2019)172[arXiv:1812.08163 [hep-ph]]

94. J. Aebischer, J. Kumar, P. Stangl, D.M. Straub, Eur. Phys. J. C 79(6), 509 (2019). https://doi.org/10.1140/epjc/s10052-019-6977-z [arXiv:1810.07698 [hep-ph]]. Version 1.3 available at https://github.com/smelli/smelli/tree/v1.3.0

95. E.E. Jenkins, A.V. Manohar, M. Trott, JHEP 01, 035 (2014). https://doi.org/10.1007/JHEP01(2014)035 [arXiv:1310.4838 [hep-ph]]

96. R. Alonso, E.E. Jenkins, A.V. Manohar, M. Trott, JHEP 04, 159 (2014). https://doi.org/10.1007/JHEP04(2014)159 [arXiv:1312.2014 [hep-ph]]

97. G. Buchalla, O. Cata, G. D'Ambrosio, Eur. Phys. J. C 74(3), 2798 (2014). https://doi.org/10.1140/epjc/s10052-014-2798-2 [arXiv:1310.2574 [hep-ph]]

98. K.A. Olive et al., [Particle Data Group], Chin. Phys. C 38, 090001 (2014). https://doi.org/10.1088/1674-1137/38/9/090001

99. D.M. Straub, [arXiv:1810.08132 [hep-ph]]. Version 1.5 available at https://github.com/flav-io/flavio/tree/v1.5.0

100. S. Aoki et al. [Flavour Lattice Averaging Group], Eur. Phys. J. C 80(2), 113 (2020). https://doi.org/10.1140/epjc/s10052-019-7354-7 [arXiv:1902.08191 [hep-lat]]

101. C. Patrignani et al. [Particle Data Group], Chin. Phys. C 40(10), 100001 (2016). https://doi.org/10.1088/1674-1137/40/10/100001

102. S. Aoki et al., Eur. Phys. J. C 77(2), 112 (2017). https://doi.org/10.1140/epjc/s10052-016-4509-7 [arXiv:1607.00299 [hep-lat]]

103. A. Bazavov et al. [Fermilab Lattice, MILC and TUMQCD], Phys. Rev. D 98(5), 054517 (2018). https://doi.org/10.1103/PhysRevD.98.054517 [arXiv:1802.04248 [hep-lat]]

104. . R. Aaij et al. [LHCb Collaboration], Phys. Rev. Lett. 125(1), 011802 (2020). https://doi.org/10.1103/PhysRevLett.125.011802 [arXiv:2003.04831 [hep-ex]]

105. R. Aaij et al., [LHCb Collaboration], JHEP 12, 081 (2020). https://doi.org/10.1007/JHEP12(2020)081 [arXiv:2010.06011 [hep-ex]]

106. [LHCb Collaboration], LHCb-CONF-2020-002

107. P. Janot, S. Jadach, Phys. Lett. B 803, 135319 (2020). https://doi.org/10.1016/j.physletb.2020.135319 [arXiv:1912.02067 [hep-ph]]

108. S. Descotes-Genon, L. Hofer, J. Matias, J. Virto, JHEP 06, 092 (2016). https://doi.org/10.1007/JHEP06(2016)092 [arXiv:1510.04239 [hep-ph]]

109. B. Capdevila, U. Laa, G. Valencia, Eur. Phys. J. C 79(6), 462 (2019). https://doi.org/10.1140/epjc/s10052-019-6944-8 [arXiv:1811.10793 [hep-ph]]

110. G. D'Amico, M. Nardecchia, P. Panci, F. Sannino, A. Strumia, R. Torre, A. Urbano, JHEP 09, 010 (2017). https://doi.org/10.1007/JHEP09(2017)010 [arXiv: 1704.05438 [hep-ph]]

111. B. Capdevila, A. Crivellin, S. Descotes-Genon, L. Hofer, J. Matias, Phys. Rev. Lett. 120(18), 181802 (2018). https://doi.org/10.1103/PhysRevLett. 120.181802 [arXiv:1712.01919 [hep-ph]]

112. F. Feruglio, PoS BEAUTY 2018, 029 (2018). https://doi.org/10.22323/1.326.0029 [arXiv:1808.01502 [hep-ph]]

113. D.A. Faroughy, A. Greljo, J.F. Kamenik, Phys. Lett. B 764, 126-134 (2017). https://doi.org/10.1016/j.physletb.2016.11.011 [arXiv:1609.07138 [hep$\mathrm{ph}] \mathrm{l}$

114. A. Greljo, J. Martin Camalich, J.D. Ruiz-Álvarez, Phys. Rev. Lett. 122(13), 131803 (2019). https://doi.org/10.1103/PhysRevLett.122.131803 [arXiv:1811.07920 [hep-ph]]

115. F. Feruglio, P. Paradisi, A. Pattori, Phys. Rev. Lett. 118(1), 011801 (2017). https://doi.org/10.1103/PhysRevLett.118.011801 [arXiv:1606.00524 [hep$\mathrm{ph}]]$

116. F. del Aguila, J. de Blas, M. Perez-Victoria, JHEP 1009, 033 (2010). https://doi.org/10.1007/JHEP09(2010)033 [arXiv:1005.3998 [hep-ph]] 